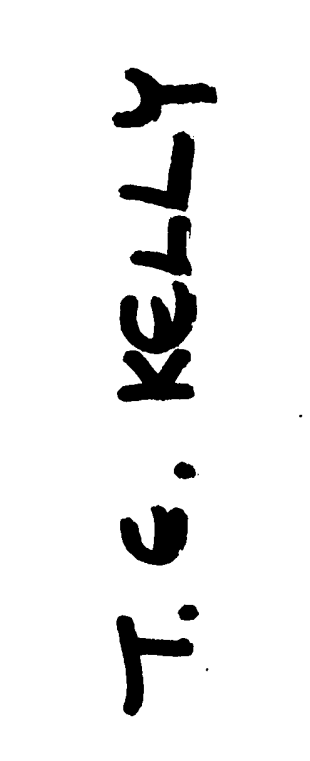




\section{UNITED STATES \\ DEPARTMENT OF THE INTERIOR \\ GEOLOGICAL SURVEY \\ Albuquerque, New Mexico}

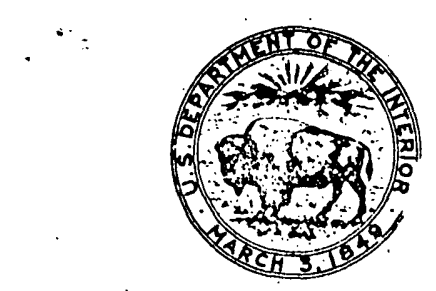

\section{SUMMARY OF GROUND-WATER DATA, POST HEADQUARTERS AND ADJACENT AREAS, WHITE SANDS MISSILE RANGE}

By

T. E. Kelly

\section{Open-file report}

Prepared by the U.S. Geological Survey, in cooperation with

White Sands Missile Range

March 1973 


\section{CONTENTS}

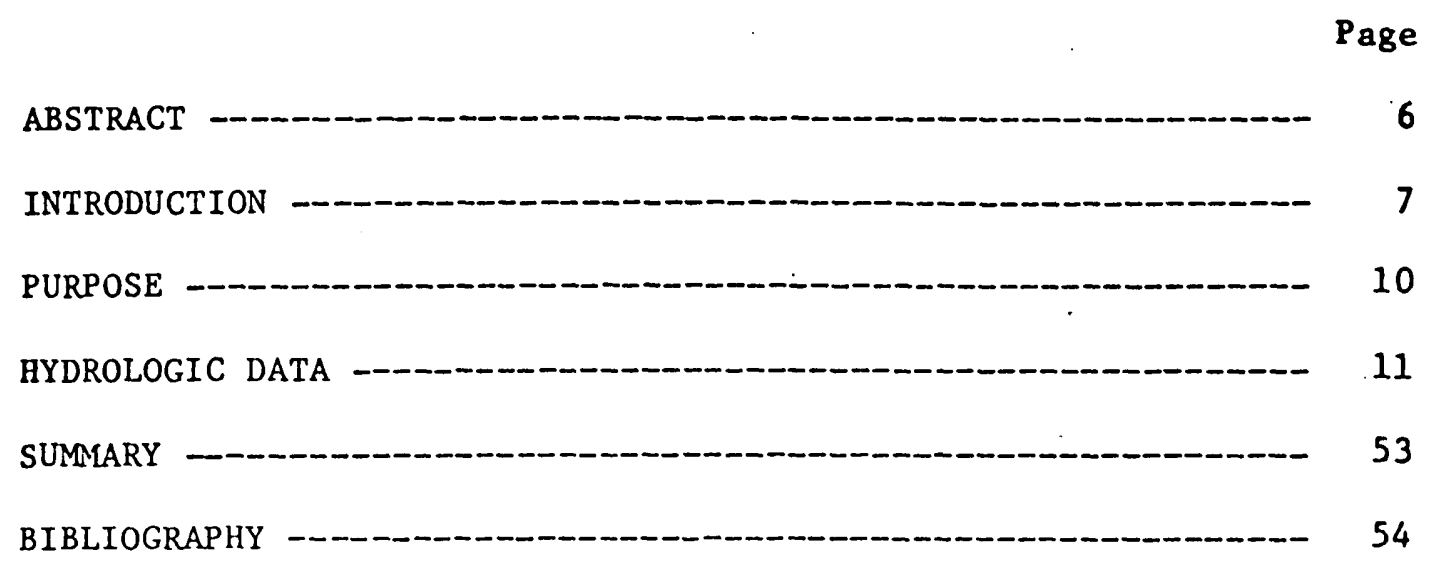




\section{ILLUSTRATIONS}

Page

Figure 1.--Index map - 8

2-3.- -Map showing:

2.--Percentage of sand between 500 and 1,000 feet --- 20

3.--Location of rain gages and surface-water

stations

4-11.--Hydrograph of:

4.--Main Gate well and monthly pumpage figures for well field - 23

5.--Test wells $\mathrm{T}-4, \mathrm{~T}-5$, and $\mathrm{T}-6$

6.--Test wells $\mathrm{T}-7$ and $\mathrm{T}-8$

7.--Test wells $\mathrm{T}-9, \mathrm{~T}-10$, and $\mathrm{T}-11$

8.--Test we11s $\mathrm{T}-12, \mathrm{~T}-13$, and $\mathrm{T}-14$

9.--Test wells $\mathrm{T}-15, \mathrm{~T}-16, \mathrm{~T}-17$, and $\mathrm{T}-18 \ldots 29$

10.--Test wells SMR-2, SMR-3, and SMR-4 30

11.--Gregg well and HTA windmill 31

12-20.--Graph showing:

12.- - Annual pumpage and hydrograph of supply well 10A- 32

13.--Annual pumpage and hydrograph of supply well $11-33$

14.--Annual pumpage and hydrograph of supply well $13-34$

15.--Annual pumpage and hydrograph of supply we11 $15-35$

16.--Annual pumpage and hydrograph of supply well $16-36$

17.--Annual pumpage and hydrograph of supply well $17-37$

18.--Annual pumpage and hydrograph of supply well $18-38$ 


\section{ILLUSTRATIONS - Concluded}

Page

Figure 19.--Annual pumpage and hydrograph of supply well $19-39$ 20.--Annual pumpage and hydrograph of supply well $20-40$ 21.--Hydrograph of supply wells HTA-1 and SMR-1 22.--Hydrograph of old supply wells 12 and $14 \ldots$ 23.--Contour map of water table, 1949

24.--Contour map of water table, 1964 25.--Map showing water-level change between 1949 and 1964 26.--Contour map of water table in Post Headquarters area, July 1972

27.--Map showing water-level change between

1964 and 1972

28.--Map showing water-level change between 1949 and 1972 50

29.--Cross sections of the Post Headquarters area --- 51 30.--Map showing configuration of base of aquifer ---- In pocket 31.--Map showing thickness of fresh water 32.--Contour map of water table, July 1972 33. - Map showing chemical quality of water samples from selected wells In pocket 


\section{TABLES}

Page

Table 1.--Record of wells and test holes in

Post Headquarters and adjacent areas 12

2.--Hydrologic characteristics of the aquifer in the vicinity of the supply and test wells

3.--Specific capacity of supply wells 


\title{
SUMMARY OF GROUND-WATER DATA, POST HEADQUARTERS AND ADJACENT AREAS, WHITE SANDS MISSILE RANGE \\ By
}

\author{
T. E. Ke11y
}

ABSTRACT

Geohydrologic data have been obtained from more than 100 wells and test holes that have been drilled in the Post Headquarters and adjacent areas of White Sands Missile Range. Observation-well data show that, in general, a continuous decline of the water table has occurred in the vicinity of the well field since production began in 1949. Approximately 40,000 acre-feet of water has been produced from the aquifer to date (1972).

A series of maps are presented which show the changes that have occurred in the well field as the result of development. 


\section{INTRODUCTION}

\section{Since 1953 a nearly continuous program of water-resources}

Investigations has been conducted on White Sands Missile Range by the U.S. Geological Survey through the cooperation of the Facilities Engineering Directorate, White Sands Missile Range. These Investigations have alded in the development and utilization of the groundwater resources, as well as contributing to the general knowledge of the geologic and hydrologic environment of the Missile Range. White Sands Missile Range is located in the Tularosa Basin, about 25 miles east of Las Cruces, N. Mex., and approximately 40 miles north of El Paso, Tex. (fig. 1). The area included in this study encompasses approximately 300 square miles and includes the Post Headquarters and adjacent areas. The Post Headquarters is situated in a reentrant formed by the junction of the San Andres, San Augustin, and Organ Mountains (fig. 1.). The eastern limit of the study area was arbitrarily set at approximately 15 miles east of the mountain axis. Where data were available, the area of investigation was extended to make optimum use of the data.

The central part of the Tularosa Basin east of the Post Headquarters contains saline water. Of particular significance to the ground-water resources of the area is the reentrant of the basin in the bordering mountain front. Seemingly this reentrant has entrapped a larger quantity of fresh water than commonly occurs along the linear mountain front, and it is here that the major water development has occurred. 
R.IE. R.2E. R.3E. R.4E R.5E. R.6E. R.7E. R.8E.

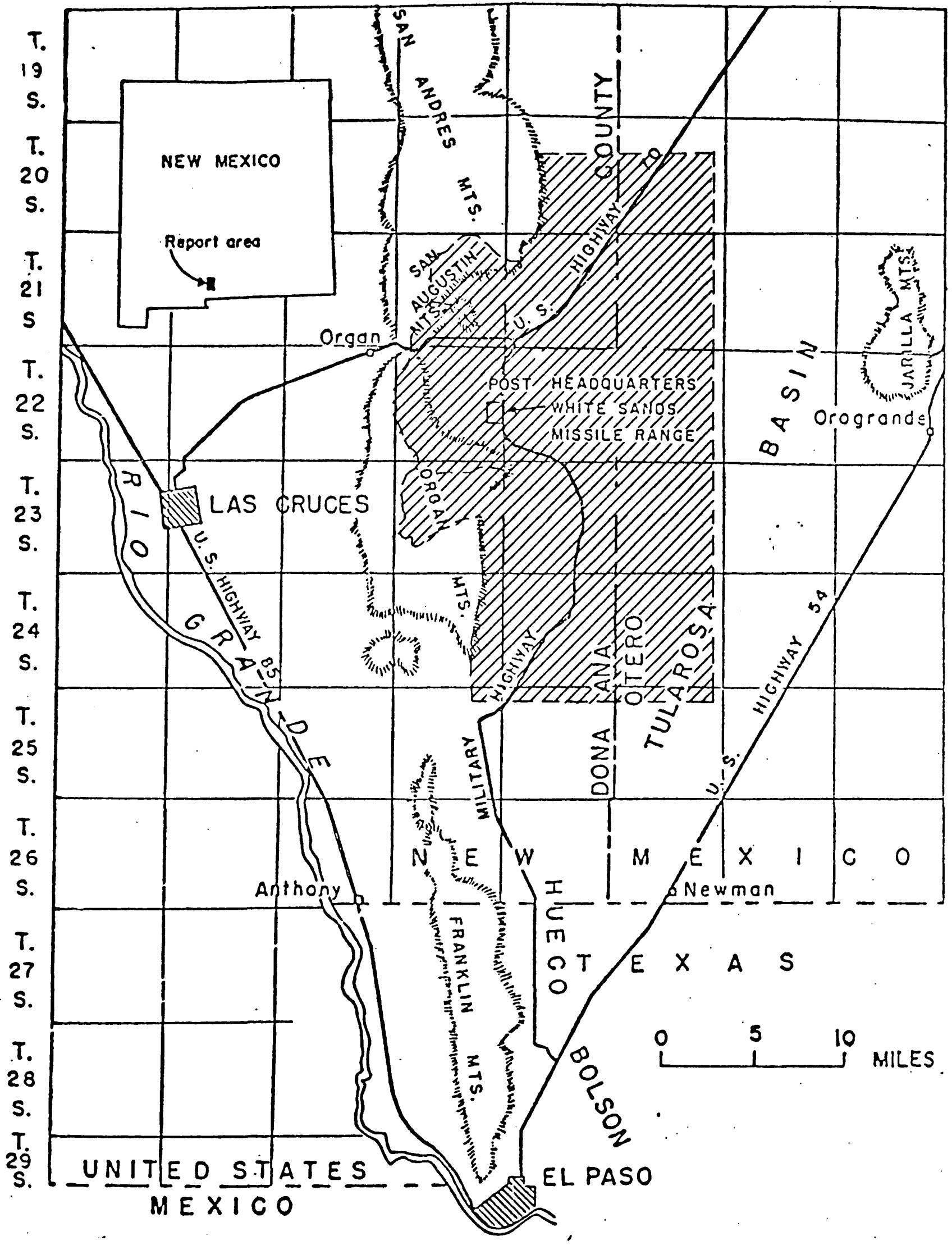

Figure 1.--Index map. 
W1th the exception of a few domestic and stock wells drilled by ranchers before establishment of the Missile Range, no groundwater had been developed in the area prior to the mid-1940's. By 1948, nine supply wells had been drilled approximately 2 miles southeast of the Post Headquarters. All but one of these wells were less than 400 feet deep; the principal water-bearing deposit was very fine sand. These wells were grouped in a tight cluster, which probably resulted in significant well interference. Consequently, this well field proved unsatisfactory and all wells were abandoned by 1953 .

Well 10 was the first supply well drilled in the Post Headquarters area; this well was completed in August 1948. Currently (1972) the total production for White Sands Missile Range is withdrawn from nine wells, excluding a few small-capacity wells that are located in outlying areas.

Eighteen test holes have been drilled in the Post Headquarters area. These were completed as observation wells to monitor waterlevel changes and water quality. In addition, five exploratory holes were drilled in the Small Missile Range north of the Post Headquarters. One of these wells (SMR-1) was completed as a supply well; three are used as observation wells. SMR-5 failed to penetrate potable water and was abandoned. Two wells were drilled in the Hazardous Test Area.

A drilling program was started in 1971 to furnish additional control for mapping the water table and water quality. A total of 57 boreholes were drilled and logged. Water-level measurements were made in each borehole and water samples were collected for chemical analysis. Approximately 15 of these boreholes are being monitored to supplement the existing observation-well network. 


\section{PURPOSE}

A large number of geologic and (or) hydrologic investigations have been made in the vicinity of White Sands Missile Range. Although each of these studies contributed to the geohydrologic knowledge of the region, little has been done to combine these data. These reports are listed in the bibllography of this report.

The possibility of saline-water encroachment from the central part of the Tularosa Basin into the Post Headquarters well field has long been recognized as a major threat to the water supply. To date, no significant change has been noted in the chemical quality of the water in any of the wells. Continued water use may result in deterioration of water quality. The purpose of this report is to summarize geohydrologic data available in the Post Headquarters and adjacent areas so that these data can be used in a forthcoming report which will evaluate the effects of ground-water withdrawal from the freshwater unft. Of primary concern is the need for reasonable estimates of future pumpage from the system and a long range plan for economical, systematic development of these natural resources. 


\section{HYDROLOGIC DATA}

More than 100 wells and test holes have been drilled within the profect area (table 1). These wells provide the data necessary to evaluate the water resources. Water-level measurements have been made in most of the wells. Field specific-conductance measurements of the water from many wells have been obtained.

The hydrologic characteristics of many of these wells are given in table 2. These data show a wide range in values, which may be due to well construction, or to variations in lithologic characteristics of the aquifer. Specific capacity of the supply wells, which is a function of well production, is given in table 3 . These data may be more useful in appraising the aquifer than the transmissivities listed in table 2. In general, the transmissivity is based on a one-well aquifer test made immediately after construction of the well; the specific capacities, though somewhat variable, are based on prolonged intervals of pumping after the well had been developed to its fullest capacity. 
Table 1.-Record of wells and test holes in Post Headquarters and adjacent areas

\begin{tabular}{|c|c|c|c|c|c|c|c|c|c|c|c|c|}
\hline socotsen & mil w. & 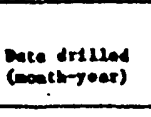 & $\begin{array}{c}\text { Deped } \\
\text { Arillod } \\
\text { (foet) }\end{array}$ & $\begin{array}{l}\text { Toenal } \\
\text { depen } \\
\text { (loet) }\end{array}$ & 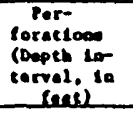 & $\begin{array}{l}\text { Meres } \\
\text { Dopth } \\
\text { below } \\
\text { ourfoce } \\
\text { (feet) }\end{array}$ & 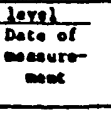 & 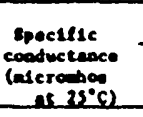 & $\begin{array}{l}\text { Alstsy } \\
\text { Leod } \\
\text { ourfoces } \\
\text { (lees) }\end{array}$ & $\begin{array}{c}\text { Wecter } \\
\text { lovel } \\
\text { (eese) }\end{array}$ & & merts \\
\hline 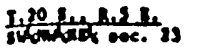 & $-\infty$ & $5-11$ & 223 & 1910 & $169-191$ & 4.25 & $2-0-12$ & $+0,000$ & 3.850 .6 & J.e4s.25 & & - \\
\hline smatean coce. is & $\operatorname{man}-3$ & $2-61$ & 1.010 & 1.000 & $120-90$ & 206.26 & $-4-12$ & $\operatorname{ces}$ & 4.277 .1 & J.8n.s4 & & - \\
\hline onduladet ece. is & $-\infty$ & -11 & 210 & 232 & $150-144$ & $d n$ & - & - & 3.905 .2 & - & & - \\
\hline 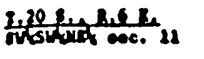 & $2-30$ & $s-n$ & 223 & 211 & $205-200$ & 279.33 & $4-19-72$ & +1.000 & 3.244 .0 & 3.054 .61 & & - \\
\hline arocotian sec. It & -n & 511 & 240 & 280 & $\begin{array}{l}100-100 \\
220-200\end{array}$ & 222.03 & $4-19-12$ & +8.000 & $3,264.0^{\circ}$ & 3.241 .05 & & - \\
\hline 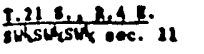 & Wimbilli & - & - & - & - & 4.84 & $\leftarrow n$ & - & 3.350 & $3,303.06$ & & - \\
\hline muntistat coe. 23 & ura-1 & $20-60$ & 130 & 130 & - & 20.60 & $22-26-69$ & 610 & 3.520 & 3.441 .31 & - & - \\
\hline 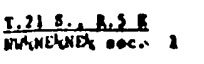 & $-x$ & $1-12$ & 200 & 281 & - & 225.84 & $2-0.12$ & 390 & $\begin{array}{l}39.3 \\
3.974\end{array}$ & $3,48.06$ & & - \\
\hline mustesty e.c. 2 & -20 & $s-n$ & 225 & 214 & $\begin{array}{l}125-165 \\
265-190\end{array}$ & 239.51 & $2-22$ & $m$ & $3,577.8$ & $3,836.20$ & & - \\
\hline SEXSWYsEt Cac. 14 & sen-s & $12-67$ & $\cos$ & 166 & $249-666$ & 108.6 & $12-24-72$ & 2.200 & 3.050 & - & Pluesed it & I aboodonod \\
\hline Intsurndet a.e. 16 & $\max 1$ & $6-\infty$ & 100. & 473 & $286-473$ & 287.50 & $2-20-12$ & ms & 4.200 .0 & 3.912 .42 & & - \\
\hline sereristset coc. 17 & $\operatorname{sen}-2$ & $-\infty$ & iss & 347 & $\begin{array}{l}295-588 \\
608-715\end{array}$ & 311.30 & $2-20-12$ & 181 & 4.140 & 3.028 .20 & & - \\
\hline monxint eac. 20 & $2-33$ & $5-11$ & sos & - & $260-290$ & en & - & - & 4.411 .8 & - & plused a & abaodocod \\
\hline SEYSWhSW eec. 20 & $\operatorname{sen}-4$ & $22-61$ & 1.016 & 300 & $470-570$ & 278.67 & $6-2-72$ & 911 & 4.290 & $3,021.33$ & & - \\
\hline SEYSWMANA cac. 23 & $2-18$ & $s-11$ & 260 & 218 & $100-220$ & 204.13 & $4-19-12$ & 3.500 & $3,940.0$ & $3,835.85$ & & - \\
\hline ovestrstry sec. 21 & $2-46$ & $1-72$ & 250 & 245 & $220-250$ & 233.58 & $2-012$ & 650 & 3.990 & 3.856 .42 & & - \\
\hline marsexset eec. 30 & $2-32$ & $5-12$ & 399 & 399 & $351-397$ & $\Delta r y$ & - & - & 4.343 .2 & $\cdot-$ & & - \\
\hline MEWNEWIEK e.e. 32 & $7-13$ & $2-67$ & 1.110 & 110 & $285-702$ & 209.26 & $6-7-72$ & 490 & 1.056 .6 & 1.841 .36 & & - \\
\hline KeXsEtwat sec. JJ & $|-1\rangle$ & $5-11$ & 284 & 243 & $230-275$ & 109.19 & $2 \cdot-n$ & 100 & $3,967.0$ & $3,837.01$ & & - \\
\hline sthmback eac. 3s & -16 & $4 n$ & 320 & 237 & $235-245$ & 207.49 & $2-8-12$ & 660 & 3.943 .6 & $3,836.11$ & & - \\
\hline 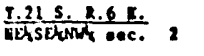 & $\rightarrow \infty$ & $2-11$ & 230 & 211 & $23 x-121$ & 156.15 & $4-19-12$ & $+1,000$ & 3.985 & $3,028.85$ & & - \\
\hline sebritsin eac. 17 & $2-27$ & $4-71$ & 200 & 374 & $263-167$ & 220.01 & $4-19-72$ & $+8,000$ & $3,945.8$ & 3.825 .73 & & - \\
\hline IE'SS BCWNA .0e. 26 & $2-40$ & $5-11$ & 237 & 223 & $\begin{array}{l}258-165 \\
183-194\end{array}$ & dry & - & - & 4,002 & - & & - \\
\hline SEhmarat eac. 32 & -26 & $4-11$ & 210 & 189 & $180-285$ & 139.95 & $4-19-72$ & 3.000 & 3.939 .6 & $3,819.63$ & & - \\
\hline 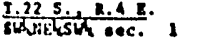 & -26 & $1-72$ & 240 & 299 & $210-240$ & 215.36 & $2-12$ & 610 & 4.478 & 4.262 .64 & & - \\
\hline mistaset sec. 1 & I-9 & $2-66$ & 398 & 398 & $338-395$ & 389.30 & $6-212$ & 222 & 4.410 .3 & 4.020 .00 & & - \\
\hline setsetset aec. 1 & $r-2$ & $(-53$ & 1.004 & 450 & $350-450$ & 399.23 & $7-31-53$ & 409 & 4.320 & $3,820.17$ & Abendosed & $2-62$ \\
\hline SEYSELNET EaC. II &,$t-0$ & $2-6$ & 1,096 & 1.060 & $374-1,040$ & 311.04 & $6-272$ & 200 & 4.441 .7 & 3.069 .06 & & - \\
\hline SEtsEtsLY .ec. 11 & -37 & $1-72$ & 340 & 321 & $510-340$ & 418.42 & $2-12$ & 460 & 4,443 & 4.024 .38 & & - \\
\hline manmatset aec. 11 & -22 & $2-72$ & 200 & 209 & $270-300$ & 242.40 & $2-2-12$ & 360 & 4,471 & 4.234 .60 & Deectroyed & $6-12$ \\
\hline BETEESEK e.c. 11 & $2-42$ & $1-72$ & 320 & 313 & $490-320$ & 384.14 & -412 & 200 & 4.373 & $3,900.66$ & & - \\
\hline Etenninder eac. 12 & $8 n-20$ & $1-65$ & 42 & 42 & 462-838 & 501.13 & $1-21-12$ & 100 & 4.354 & $3,832.21$ & & - \\
\hline Ieburset .ec. 12 & su-1s & $6-6$ & 203 & $\infty 00$ & $400-100$ & 41.08 & $1-27-12$ & 2so & 4.294 & 3.052 .95 & & - \\
\hline setsurset .ec. 22 & $0-10$ & $5-64$ & 100 & $\infty$ & $400-\infty 00$ & 417.52 & $1-27-72$ & 302 & 4,264 & 3.846 .48 & & - \\
\hline mrsmank sec. 13 & $(n-1)$ & $-\infty$ & 900 & 900 & $436-886$ & 431.85 & $1-27-72$ & 312 & 4.260 & 3.828 .15 & & - \\
\hline meonstrat aec. IJ & (1)-13 & est & sus & 334 & $\begin{array}{l}313-393 \\
470-534\end{array}$ & $\$ 12$ & $1-21-72$ & 331 & 4.330 .3 & 4.018 .3 & & - \\
\hline smovetret sec. 13 & $x-2$ & 2-53 & 1.000 & 400 & $300-400$ & 337.96 & $2-29-33$ & sos & $4,262.6$ & 3.924 .62 & Abeodoned & $12-60$ \\
\hline rammate nec. 13 & $n+24$ & $2-33$ & 1.005 & 110 & $310-810$ & $\$ 96.20$ & $+9-71$ & 321 & 4.290 .3 & $3,094.10$ & Pluesed & \\
\hline IEtretset .ec. 13 & su-1s & $2-34$ & 1.010 & 820 & $350-220$ & 421 & $1-21-72$ & 323 & 4.261 .3 & $3,840.3$ & & - \\
\hline netsmiset coc. 13 & ow-16 & $2-54$ & 1.020 & 900 & $370-186$ & 433 & $\mid-27-72$ & 3 & 4.270 & $3,837.0$ & . & - \\
\hline shrsumenm e.ec. 14 & $I \rightarrow$ & $11-60$ & s1s & s1s & $285-409$ & 208.18 & $6-27$ & (3) & 4.507 & 4.298 .02 & & - \\
\hline Itssumm nec. 16 & -20 & $2-11$ & ४47 & 426 & $410-440$ & 333.69 & $2-12$ & 460 & 4,446 & 4.112 .31 & & - \\
\hline munerext e.ec. 14 & $x-3$ & $2-33$ & ens & 450 & $350-4502$ & 381.25 & $(-453$ & 610 & 4.442 .4 & $0,061.15$ & Nandoned & $1-61$ \\
\hline
\end{tabular}


Table 1.--Record of wells and test holes in Post Headquarters and adjacent areas - Continued

\begin{tabular}{|c|c|c|c|c|c|c|c|c|c|c|c|}
\hline Loceltion & well no. & $\begin{array}{l}\text { nate drilled } \\
\text { (month-year) }\end{array}$ & $\begin{array}{l}\text { Depth } \\
\text { drilled } \\
\text { (feet) }\end{array}$ & $\begin{array}{l}\text { Totel } \\
\text { depth } \\
\text { (foet) }\end{array}$ & $\begin{array}{l}\text { Per- } \\
\text { foratione } \\
\text { (Depth 1n- } \\
\text { lerval, 10 } \\
\text { (eest) } \\
\end{array}$ & $\begin{array}{l}\text { Nater } \\
\text { Depith } \\
\text { helow } \\
\text { curface } \\
\text { (feet) }\end{array}$ & $\begin{array}{l}\frac{\text { level }}{\text { Date of }} \\
\text { meoure- } \\
\text { ment }\end{array}$ & 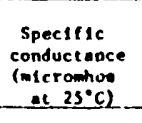 & $\begin{array}{c}\text { Alt litu } \\
\text { Land } \\
\text { ourfoce } \\
\text { (feet) }\end{array}$ & $\begin{array}{l}\text { ude } \\
\text { Wacer } \\
\text { level } \\
\text { (feet) }\end{array}$ & Reanerike \\
\hline 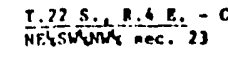 & $\begin{array}{l}\text { concluded } \\
3-216\end{array}$ & $1-12$ & 195 & 184 & $164-184$ & dey & - & - & 4.411 & - & - \\
\hline 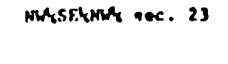 & -21e & $3-71$ & 394 & - & $\begin{array}{l}278-298 \\
328-338\end{array}$ & dry & - & - & - & - & Plurged o shondoned \\
\hline SAtstyset eec. 2] & $T-12$ & $3-67$ & 2.000 & 1.820 & $1.630-1,810$ & 211.19 & $6-8-72$ & 410 & 4.334 .6 & 4.123 .41 & $\begin{array}{l}\text { Bridged at } 220 \mathrm{ft} \text { by } \\
\text { vandalo }\end{array}$ \\
\hline NELWhermA sec. 24 & sw-11 & $3-30$ & 323 & 500 & $380-490$ & 219 & $1-27-72$ & 321 & 4.333 & 4.042 & - \\
\hline NELMMANEY BeC. 24 & $s W-10$ & 8.48 & sos & 494 & $264-294$ & 358 & $8-12-53$ & 337 & 4.271 .3 & 3.913 .3 & Abandoned $7-63$ \\
\hline Nobmrwet nec. 24 & $s \dot{H}-104$ & $1-63$ & 825 & $\cos$ & $405-805$ & $\$ 20.70$ & $1-27-72$ & 321 & 4,273 & 3.852 .30 & - \\
\hline NEZWNELNES ace. 24 & Maln Gate & $6-59$ & 440 & 431 & $388-438$ & 405.71 & $6-8-12$ & 361 & 4.252 .9 & $3,841.19$ & - \\
\hline 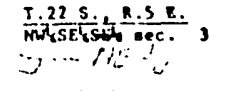 & $8-14$ & $3-71$ & 263 & 209 & $\begin{array}{l}180-220 \\
240-258\end{array}$ & 110.94 & $3-8-72$ & 800 & $3,942.5$ & $3,831.56$ & - \\
\hline NF'SEXNAS ReC. S & $8-15$ & $4-71$ & 255 & 225 & $200-220$ & 168.15 & $3-9-72$ & 500 & 4.037 & - & - \\
\hline swamirsuty oec. S & $T-10$ & $9-66$ & 1,363 & sss & $370-545$ & 260.49 & $6-7-72$ & 605 & 4.159 .8 & $3.69 n .31$ & - \\
\hline SF.4SWHALt sec. 6 & $8-24$ & $4-71$ & 260 & 253 & $180-260$ & dry & - & $\cdots$ & 4.331 .1 & - & - \\
\hline SWESEMAN sec. 6 & $8-25$ & $4-71$ & 280 & 264 & $260-270$ & dry & - & - & 4.253 .8 & - & - \\
\hline MANE'SHA oec. 6 & $8-53$ & $12-71$ & 400 & 400 & $370-400$ & 391.41 & $3-9-72$ & 120 & 4.260 & 3.868 .59 & - \\
\hline NE''SSELWEXX sec. 1 & $B-50$ & $12-11$ & 350 & 349 & $320-350$ & 293.10 & $3-8-72$ & 440 & 4,110 & $3,876.90$ & - \\
\hline NE'SEXSWW sec. 1 & $T-7$ & $8-63$ & 1.000 & 1.000 & $326-966$ & 362.49 & $6-6-72$ & 376 & 4,185 & 3.022 .51 & - \\
\hline MRrSEE'tont sec. 8 & $8-13$ & $4-11$ & 315 & 287 & $250-285$ & 233.46 & $3-8-72$ & 400 & 4.101 & 3.861 .56 & - \\
\hline SFiłsinsm sec. & $8-41$ & $12-71$ & 350 & 338 & $320-350$ & 267.60 & $3-8-12$ & 355 & 4,116 & $3,846.40$ & - \\
\hline SHAminim sec. & $8-49$ & $12-71$ & 200 & 200 & $170-200$ & 196.32 & $3-8-12$ & - & 4.040 & $3,843.88$ & Cluster 1 \\
\hline strumant sec. 9 & $8-52$ & $\cdot 12-71$ & 350 & 336 & $320-350$ & 207.15 & $3-8-12$ & $35 s$ & 4,061 & 3.833 .85 & Do. \\
\hline strumbars sec. 9 & $8-53$ & $1-12$ & 300 & $\stackrel{473}{\dddot{2}}$ & $470-500$ & 212.85 & $3-8-72$ & 320 & 4.040 & 3.821 .15 & Do. \\
\hline MrNE'SNIX nec. 15 & $T-16$ & $10-67$ & 6.015 & 370 & $210-360$ & 133.15 & $6-6-72$ & 683 & 3,950 & 3.826 .85 & - \\
\hline 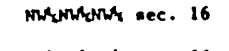 & $B-23)$ & $12-71$ & 250 & $250-$ & $220-250\}$ & 222.95 & $3-8-72$ & 370 & 4.050 & 3.827 .05 & Cluater 2 \\
\hline Mrentrwh sec. 16 & $=-35$ ? & $12-71$ & 400 & 388 & $370-400$ & 216.42 & $3-8-72$ & 340 & 4,046 & 3.029 .58 & Do. \\
\hline Nherresshs aec. 16 & $8-54 J$ & $12-71$ & 500 & 437. & ה 470-500 & 228.90 & $3-8-72$ & 293 & 4.067 & 3.818 .10 & Do. \\
\hline 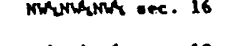 & $T-4$ & $6-33$ & 1.000 & 400 & $300-400$ & 225.32 & $6-7-12$ & 313 & 4.051 .3 & $3 . n 25.98$ & - \\
\hline 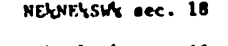 & $8-12$ & $4-11$ & 408 & 309 & $355-365$ & 283.96 & $3-9-12$ & - & 4.152 .6 & $3,868.64$ & - \\
\hline SFWriksex sec. 19 & $2-10$ & $4-11$ & 420 & 385 & $\begin{array}{l}370-373 \\
390-395\end{array}$ & 300.19 & $3-9-12$ & cos & 4.169. & 3.869 .61 & - \\
\hline menermes eec. 20 & T-5 & $7-53$ & 1.000 & 400 & $300-400$ & 214.02 & $6-6-72$ & 379 & $4,149.8$ & $3,815.78$ & - \\
\hline monswet nee. 21 & -9 & $3-11$ & 260 & 254 & $240-250$ & 226.60 & $3-9-72$ & 380 & $4,036.2$ & 3.009 .60 & - \\
\hline matsetsin eec. 23 & $2-1$ & $3-11$ & 233 & 205 & $195-213$ & 175.42 & $9-9-71$ & 480 & 3.976 .6 & $3,801.10$ & - \\
\hline MHasetsitk aec. 23 & $\rightarrow-8$ & $4-71$ & 330 & 291 & 290-293 & 176.25 & $3-9-12$ & 460 & $3,976.6$ & 3.800 .35 & - \\
\hline neturastar ene. 26 & $2-51$ & $2-72$ & 400 & 312 & $370-400$ & 141.90 & $3-9-72$ & 340 & 3,946 & $3,004.10$ & - \\
\hline 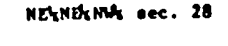 & $8-1$ & $3-11$ & 239 & 248 & $210-230$ & 193.54 & $3-8-12$ & - & 4.013 .0 & $3,821.46$ & • \\
\hline seweante eec. 28 & -2 & 4 & 260 & 256 & $220-260$ & 203.26 & $9-9-71$ & - & 4.015 .4 & $3,812.14$ & - \\
\hline wetsetom axe. 20 & -3 & $\rightarrow-\infty$ & 280 & 280 & $220-280$ & - & - & - & 4.019 .7 & - & - \\
\hline sutsturnet see. 20 & $1-4$ & $2-71$ & 260 & 232 & $220-260$. & 200.13 & $3-9-12$ & - & $4,016.6$ & 3.015 .81 & - \\
\hline netstenter oec. 29 & $T-11$ & $12-66$ & 1.800 & 180 & $306-160$ & 275.58 & $6-1-12$ & 336 & 4.081 .2 & $3,805.62$ & - \\
\hline suonnessty exc. 30 & $2-11$ & $4-71$ & 300 & 284 & 273-283 & dry & - & - & 4.220 .1 & - & - \\
\hline sewesset •ec. 30 & $8-56$ & $12-11$ & 350 & 350 & $320-330$ & 211.80 & $3-9-72$ & 440 & 4.162 & $3,936.20$ & - \\
\hline sechetset aec. 31 & Nn-9 & $6-46$ & 340 & 348 & $259-320$ & 234 & $9-10-47$ & 240 & 4.128 .0 & $3,094.0$ & - \\
\hline setswonk eec. 32 & sบ-1 & - & - & 237 & $231-257$ & dry & $(1-31-53$ & - & 4,130 & - & - \\
\hline stevourd aec. 32 & $2-5$ & $3-11$ & - & 249 & $210-249$ & 189.63 & $3-9-72$ & - & $3,996.2$ & $3,006.31$ & - \\
\hline meoneste occ. 32 & $n-2$ & - & 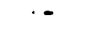 & 296 & $250-296$ & dry & $1-31-53$ & - & 4,130 & - & $\cdot$ \\
\hline
\end{tabular}


Table 1,--Record of wells and test holes in Post Headquarters and

$$
\text { adjacent areas - Concluded }
$$

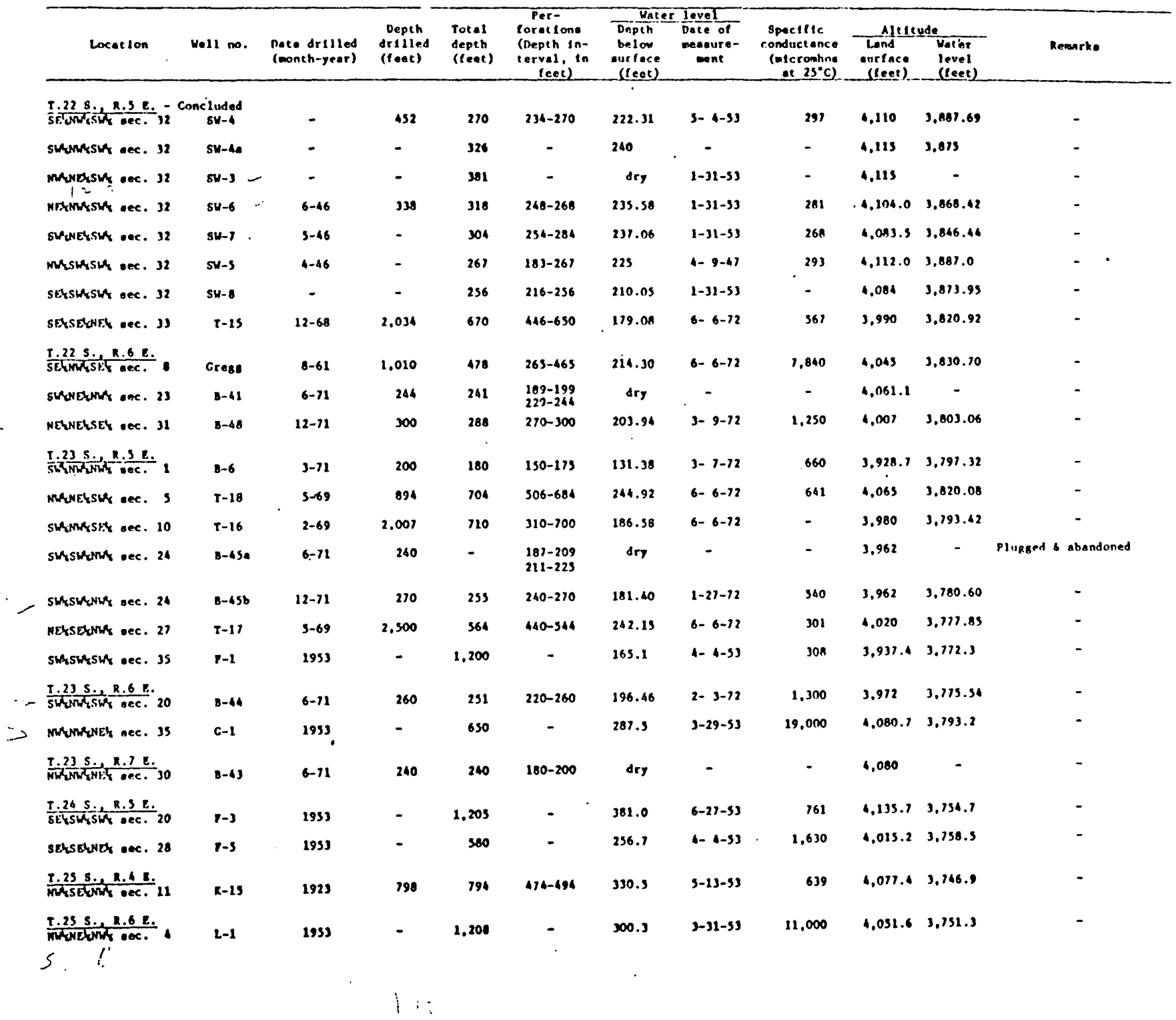


Table 2.--Hydrologic characteristics of the aquifer in the vicinity of supply and test wells

\begin{tabular}{|c|c|c|c|c|c|c|c|}
\hline Location & Well no. & $\begin{array}{l}\text { Completion } \\
\text { depth } \\
\text { (feet) }\end{array}$ & $\begin{array}{l}\text { Saturated } \\
\text { thickness } \\
\text { penetrated } \\
\text { (feet) } \\
\end{array}$ & $\begin{array}{l}\text { Apparent } \\
\text { trans- } \\
\text { missi- } \\
\text { bility } \\
\text { (gpd/ft) } \\
\end{array}$ & $\begin{array}{l}\text { Apparent } \\
\text { trans- } \\
\text { missi- } \\
\text { vity } \\
\left(\mathrm{ft}^{2} / \text { day) }\right.\end{array}$ & $\begin{array}{l}\text { Spec1fic } \\
\text { capac1ty } \\
(\mathrm{gpm} / \mathrm{ft}) \\
\end{array}$ & $\begin{array}{l}\text { Sand fractlon } \\
500-1,000 \text { feet } \\
\text { or total depth }\end{array}$ \\
\hline 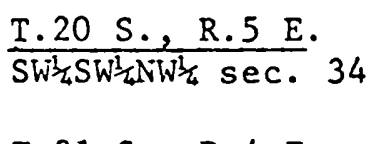 & SMR -3 & 1,000 & 703 & 350,000 & 46,900 & 96 & 52 \\
\hline 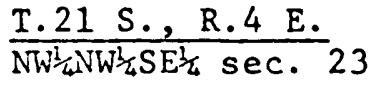 & HTA-1 & 392 & 314 & 1,700 & 228 & 1.4 & - \\
\hline$\frac{\text { T.21 S., R. } 5 \text { E. }}{\text { SE }^{\frac{1}{4} S I^{1} \frac{1}{4} \mathrm{SE}^{\frac{1}{4}} \mathrm{sec} .}} 14$ & $S M R-5$ & Plugged & 666 & no test & - & - & 70 \\
\hline$N W^{\frac{1}{4}} \mathrm{SW}^{\frac{1}{4}} \mathrm{NE} \frac{1}{4}$ sec. 16 & SMR -1 & 473 & 192 & 7,900 & 1,158 & 5.9 & - \\
\hline $\mathrm{SE}^{1} / \mathrm{NE}^{1} / \mathrm{SE} \mathrm{E}_{4}^{1} \mathrm{sec} .17$ & SMR-2 & 765 & 460 & 20,000 & 2,680 & 12.3 & - \\
\hline$S E^{-1} \frac{1}{4} S W^{\frac{1}{4}} \frac{S}{S} W^{\frac{1}{4}} \frac{1}{4}$ sec. 20 & SMR-4 & 580 & 306 & 100,000 & 13,400 & 29 & 32 \\
\hline $\begin{array}{l}N E^{-\frac{1}{4}} N^{\frac{1}{4}} \mathrm{NE}^{\frac{1}{4}} \text { sec. } 32 \\
\text { T. } 22 \text { S., R. } 4 \text { E. }\end{array}$ & $\mathrm{T}-13$ & 710 & 501 & 6,000 & 804 & 3.1 & 38 \\
\hline 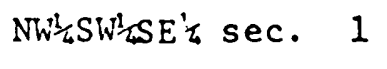 & $\mathrm{T}-9$ & 598 & 208 & 32.7 & 4.4 & .1 & 80 \\
\hline 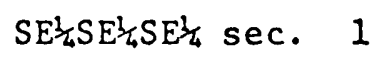 & $\mathrm{T}-1$ & 450 & 50 & no test & - & - & .56 \\
\hline SE $\frac{1}{4} \mathrm{SE}^{\frac{1}{2}} \mathrm{NE} E^{\frac{1}{4}}$ sec. 11 & $\mathrm{~T}-8$ & 895 & 342 & 1,200 & 160 & 2.0 & .52 \\
\hline SE $\frac{1}{4} W^{1} \frac{1}{4} N E^{2} \frac{1}{4}$ sec. 12 & $\mathrm{SW}-20$ & 842 & 380 & 828,000 & 111,900 & 132 & .61 \\
\hline
\end{tabular}


Table 2.--Hydrologic characterlstics of the aquifer in the vicinity of supply and test wells - Cont1nued

\begin{tabular}{|c|c|c|c|c|c|c|c|}
\hline Location & We11 no. & $\begin{array}{c}\text { Completion } \\
\text { depth } \\
\text { (feet) }\end{array}$ & $\begin{array}{l}\text { Saturated } \\
\text { thickness } \\
\text { penetrated } \\
\text { (feet) }\end{array}$ & $\begin{array}{c}\text { Apparent } \\
\text { trans- } \\
\text { missi- } \\
\text { bility } \\
\text { (gpd/ft) } \\
\end{array}$ & $\begin{array}{c}\text { Apparent } \\
\text { trans- } \\
\text { missi- } \\
\text { vity } \\
\text { (ft /day }\end{array}$ & $\begin{array}{l}\text { Specific } \\
\text { capacity } \\
(\mathrm{gpm} / \mathrm{ft}) \\
\end{array}$ & $\begin{array}{l}\text { Sand fraction } \\
500-1,000 \text { feet } \\
\text { or total depth }\end{array}$ \\
\hline 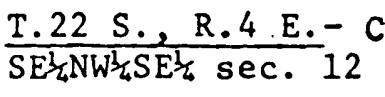 & $\begin{array}{l}\text { tinued } \\
\text { SW-19 }\end{array}$ & 800 & 391 & 79,600 & 10,666 & 44 & 45 \\
\hline 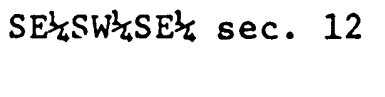 & SW-18 & 800 & $\begin{array}{l}405 \\
385\end{array}$ & $\begin{array}{l}4,780 \\
5,440\end{array}$ & $\begin{array}{l}632 \\
743\end{array}$ & $\begin{array}{l}18.1 \\
21.2\end{array}$ & 44 \\
\hline 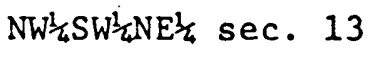 & $S W-17$ & 800 & 504 & 30,000 & 4,020 & 26.1 & 44 \\
\hline$N W^{\frac{1}{4}} N W^{\frac{1}{4}} \operatorname{SWW}_{4}^{\frac{1}{4}}$ sec. 13 & SW-13 & 534 & 234 & 5,000 & 670 & 3.5 & 64 \\
\hline SW $\frac{1}{2} N E^{\frac{1}{4}} N^{\prime} \frac{1}{4}$ sec. 13 & $\mathrm{~T}-2$ & 400 & 62 & no test & - & - & 35 \\
\hline$N W^{\frac{1}{4}} N W^{\frac{1}{4}} \mathrm{~S} E^{\frac{1}{4}}$ sec. 13 & SW-14 & 810 & 453 & 8,000 & 1,070 & 7.8 & 40 \\
\hline $\mathrm{SE}_{4}^{\frac{1}{4}} \mathrm{NE}_{4}^{\frac{1}{4} \mathrm{SE}} \frac{1}{4}$ sec. 13 & SW-15 & 820 & 482 & 28,300 & 3,790 & 18.6 & 42 \\
\hline $\mathrm{NE}^{\frac{1}{4}} \mathrm{SW}^{\frac{1}{4}} \mathrm{~S} E^{\frac{1}{4}}$ sec. 13 & SW-16 & 890 & 540 & 33,000 & 4,420 & 45.0 & 40 \\
\hline SW $\frac{1}{4} \mathrm{SW}_{2}^{\frac{1}{4}} \mathrm{NW}^{\frac{1}{4}}$ sec. 14 & $\mathrm{~T}-6$ & 515 & 311 & no test & - & 2.7 & - \\
\hline$N W^{\frac{1}{4} N W^{\prime}} \frac{1}{4} N E^{\frac{1}{4}}$ sec. 14 & $\mathrm{~T}-3$ & 450 & 69 & no test & - & - & $32 \star$ \\
\hline $\mathrm{SE}^{\frac{1}{4}} \mathrm{~N} W^{\frac{1}{4}} \mathrm{NE} \frac{1}{4}$ sec. 23 & SW-12 & 570 & 285 & 5,000 & 670 & 3.2 & 59 \\
\hline $\mathrm{SE}_{\frac{1}{4}} \mathrm{SE}_{\frac{1}{4}} \mathrm{SE}^{\frac{1}{4}}$ sec. 23 & $T-12$ & 1,820 & 1,589 & 1,360 & 182 & 1.5 & 49 \\
\hline NE⿱亠幺幺小 NW $\frac{1}{4} N W^{\frac{1}{4}}$ sec. 24 & $S W-11$ & 500 & 195 & 2,500 & 335 & 12.0 & - \\
\hline
\end{tabular}




\begin{tabular}{|c|c|c|c|c|c|c|c|}
\hline Location & We11 no. & $\begin{array}{c}\text { Completion } \\
\text { depth } \\
\text { (feet) }\end{array}$ & $\begin{array}{l}\text { Saturated } \\
\text { thickness } \\
\text { penetrated } \\
\text { (feet) }\end{array}$ & $\begin{array}{l}\text { Apparent } \\
\text { trans- } \\
\text { missi- } \\
\text { bility } \\
\text { (gpd/ft) } \\
\end{array}$ & $\begin{array}{l}\text { Apparent } \\
\text { trans- } \\
\text { missi- } \\
\text { vity } \\
\left(\mathrm{ft}^{2} / \text { day) }\right. \\
\end{array}$ & $\begin{array}{l}\text { Specific } \\
\text { capacity } \\
(\mathrm{gpm} / \mathrm{ft}) \\
\end{array}$ & $\begin{array}{l}\text { Sand fraction } \\
500-1,000 \text { feet } \\
\text { or total depth }\end{array}$ \\
\hline T:22 S., R.4 E. - & Concluded & & & & & & \\
\hline$\overline{N E^{\frac{1}{4}} N W^{\frac{1}{4}} N^{2} E^{\frac{1}{4}}}$ sec. 24 & SW-10 & 505 & 139 & 61,600 & 8,170 & 23.6 & - \\
\hline $\mathrm{NE}^{\frac{1}{4}} \mathrm{NW}_{\frac{1}{4}} \mathrm{NE}_{\frac{1}{4}}^{\frac{1}{4}}$ sec. 24 & $\mathrm{SW}-10 \mathrm{~A}$ & 805 & 404 & 114,000 & 15,300 & 37.5 & 48 \\
\hline$N E_{\frac{1}{4}} N_{E}^{\frac{1}{2}} N^{2} \frac{1}{2}$ sec. 24 & Main Gate & 430 & 73 & 17,600 & 2,360 & 11.5 & - \\
\hline$\frac{\text { T.2.2 S., R.5 E }}{\text { SW }^{\frac{1}{4} \mathrm{NW}^{\frac{1}{4}} \mathrm{~S} \mathrm{~W}^{\frac{1}{4}} \mathrm{sec} \cdot}}=$ & $T-10$ & 555 & 305 & 13,900 & 1,862 & 7 & .14 \\
\hline $\mathrm{NE}_{\frac{1}{4}}^{1} \mathrm{SE}_{\frac{1}{4}} \mathrm{SW}_{\frac{1}{4}}$ sec. 7 & $T-7$ & 1,000 & 660 & 12,000 & 1,620 & 12 & 22 \\
\hline $\mathrm{NT}^{\frac{1}{4}} \mathrm{NE}_{4}^{\frac{1}{4}} \mathrm{NE}^{\frac{1}{4}} \mathrm{sec} \cdot 15$ & $\mathrm{~T}-14$ & 370 & 239 & no test & - & - & 45 \\
\hline$N N^{1} \frac{1}{4} N W^{1} \frac{1}{4} N W^{\frac{1}{4}}$ sec. 16 & $T-4$ & 400 & 177 & no test & - & - & 39 \\
\hline$N W^{\frac{1}{2}} N W^{\frac{1}{4}} \mathrm{~N} W^{\frac{1}{2}} \frac{\mathrm{s}}{4}$ sec. 20 & $\mathrm{~T}-5$ & 400 & 130 & no test & - & - & 40 \\
\hline $\mathrm{NE}^{\frac{1}{4}} \mathrm{SW}_{\frac{1}{4}} \mathrm{NE}^{\frac{1}{4}}$ sec. 29 & $\mathrm{~T}-11$ & 800 & 521 & 12,750 & 1,698 & 6 & 35 \\
\hline $\mathrm{SE}_{\frac{1}{4}}^{\frac{1}{4}} \mathrm{SE}_{\frac{1}{4}} \mathrm{NE}^{\frac{1}{4}} \mathrm{sec} .33$ & $T-15$ & 670 & 483 & no test & - & - & 40 \\
\hline$\frac{T .22 \mathrm{~S} ., \mathrm{R} \cdot 6 \mathrm{E}}{\mathrm{SE}_{\frac{1}{4}} \mathrm{NW}^{\frac{1}{4} \mathrm{SE}} \frac{1}{4} \mathrm{sec} \cdot}{ }_{8}$ & Gregg & 500 & 270 & 19,600 & 2,626 & 12.4 & 54 \\
\hline 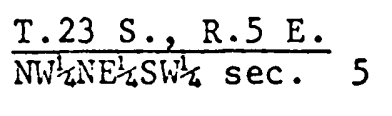 & $T-18$ & 704 & 447 & 1,150 & 254 & 1.0 & $32 \star$ \\
\hline $\mathrm{SW}^{1} \frac{1}{4} \mathrm{NW}_{\frac{1}{4}} \mathrm{SE}^{\frac{1}{4}} \frac{\mathrm{sec}}{} 10$ & $\mathrm{~T}-16$ & 710 & 523 & 37,600 & 5,040 & 10.8 & 61 \\
\hline $\mathrm{NE}^{\frac{1}{4}} \mathrm{SE}^{\frac{1}{2}} \mathrm{NW} W_{4}^{\frac{1}{4}} \mathrm{sec} .27$ & $\mathrm{~T}-17$ & 564 & 322 & 16,400 & 2,197 & 5.7 & 54 \\
\hline
\end{tabular}


Table 3.--Specific capacity of supply wells

\begin{tabular}{|c|c|c|c|c|c|c|c|c|c|c|c|c|c|c|c|c|c|c|c|c|c|c|}
\hline \multirow{2}{*}{$\begin{array}{l}\text { Well } \\
\text { number }\end{array}$} & \multicolumn{22}{|c|}{ Year } \\
\hline & 1951 & 1952 & 1953 & 1954 & 1955 & 1956 & 1957 & 1958 & 1959 & 1960 & 1961 & 1962 & 1963 & 1964 & 1965 & 1966 & 1967 & 1968 & 1969 & 1970 & 1971 & 1972 \\
\hline 10 & - & - & - & - & 17.7 & 18.7 & 10.1 & 9.6 & 10.9 & 13.1 & 11.9 & 11.7 & 12.8 & - & - & - & - & - & - & - & - & - \\
\hline $10 \mathrm{~A}$ & - & - & - & - & - & - & - & - & - & - & - & - & - & 39.6 & 44.4 & 37.5 & 90.0 & 53.3 & 51.6 & 45.8 & 47.9 & 46.8 \\
\hline 11 & - & - & - & - & - & 16.6 & 20.7 & 23.5 & 23.4 & 23.3 & 24.8 & 24.2 & 47.3 & - & 31.0 & 25.0 & 29.8 & 32.6 & 16.3 & 25.3 & 26.4 & - \\
\hline 12 & - & - & 9.7 & 12.3 & 4.8 & 8.0 & - & - & - & - & - & - & - & - & - & - & - & - & - & - & - & - \\
\hline 13 & 13.9 & - & - & - & 16.5 & 30.9 & 8.3 & 10.4 & 16.8 & 6.9 & 8.9 & 12.9 & 9.0 & 8.5 & 8.0 & 9.2 & - & 14.4 & 6.6 & 9.1 & 15.8 & 9.5 \\
\hline 14 & - & - & - & - & 13.5 & 15.9 & 13.6 & 9.6 & 31.2 & 11.7 & 11.0 & 6.6 & 7.5 & 5.9 & - & - & - & - & - & - & - & - \\
\hline 15 & - & - & - & - & 32.0 & 26.9 & 29.0 & 25.0 & 22.6 & 23.0 & 22.1 & 29.0 & 20.0 & 14.6 & 21.5 & 15.6 & 16.5 & 16.6 & 15.2 & 25.4 & 12.5 & 15.0 \\
\hline 16 & - & - & - & - & 25.7 & 30.6 & 40.8 & 43.1 & 31.0 & 26.4 & 28.4 & 35.9 & 55.5 & 41.0 & 41.6 & 46.8 & 44.0 & 46.6 & 36.8 & 38.2 & 38.5 & 47.0 \\
\hline 17 & - & - & - & - & - & - & - & - & - & - & - & 35.0 & 33.1 & 31.1 & 26.0 & 37.4 & - & 40.4 & 30.8 & 30.2 & 39.8 & 29.3 \\
\hline 18 & - & - & - & - & - & - & - & - & - & - & - & - & - & - & 11.8 & 9.2 & 14.0 & 11.1 & 8.4 & 8.9 & - & 7.6 \\
\hline 19 & - & - & - & - & & - & - & - & - & - & - & - & - & - & 62.0 & 60.6 & 51.3 & 66.7 & 50.2 & 53.3 & 62.8 & 55.3 \\
\hline 20 & - & - & - & - & - & - & - & - & - & - & - & - & - & - & 156.4 & 117.3 & - & 156.4 & 111.2 & 194.4 & 125.0 & 117.1 \\
\hline SMB-1 & - & - & - & - & - & - & - & - & - & - & - & 7.7 & 7.7 & 6.3 & 6.3 & - & - & - & - & - & - & - \\
\hline
\end{tabular}


The sand percentage was calculated from geophysical logs for the depth interval 500 to 1,000 feet (table 2 and f1g. 2). This interval was selected because in most cases it is below the water table and because various sources of information indicate that permeability significantly decreases below 1,000 feet. Therefore this interval is believed to be the major water-producing zone. Although several exceptions were noted, in general the areas having the highest sand percentages are those where transmissivity and specific capacity are greatest. The sand percentage generally decreases basinward, but nearly all supply wells are located in areas where the sand percentage exceeds 40 percent. Sixty-one percent sand was present in supply well 20; this well also has the highest transmissivity recorded in the well field (table 2). Other wells show very poor correlation between sand percentage and transmissivity.

Precipitation and runoff data are being monitored throughout the Post Headquarters area. The location of the stations are shown on figure 3. These data have been published annually (Cruz, 1972, table 5); no new data were collected as part of this investigation. 


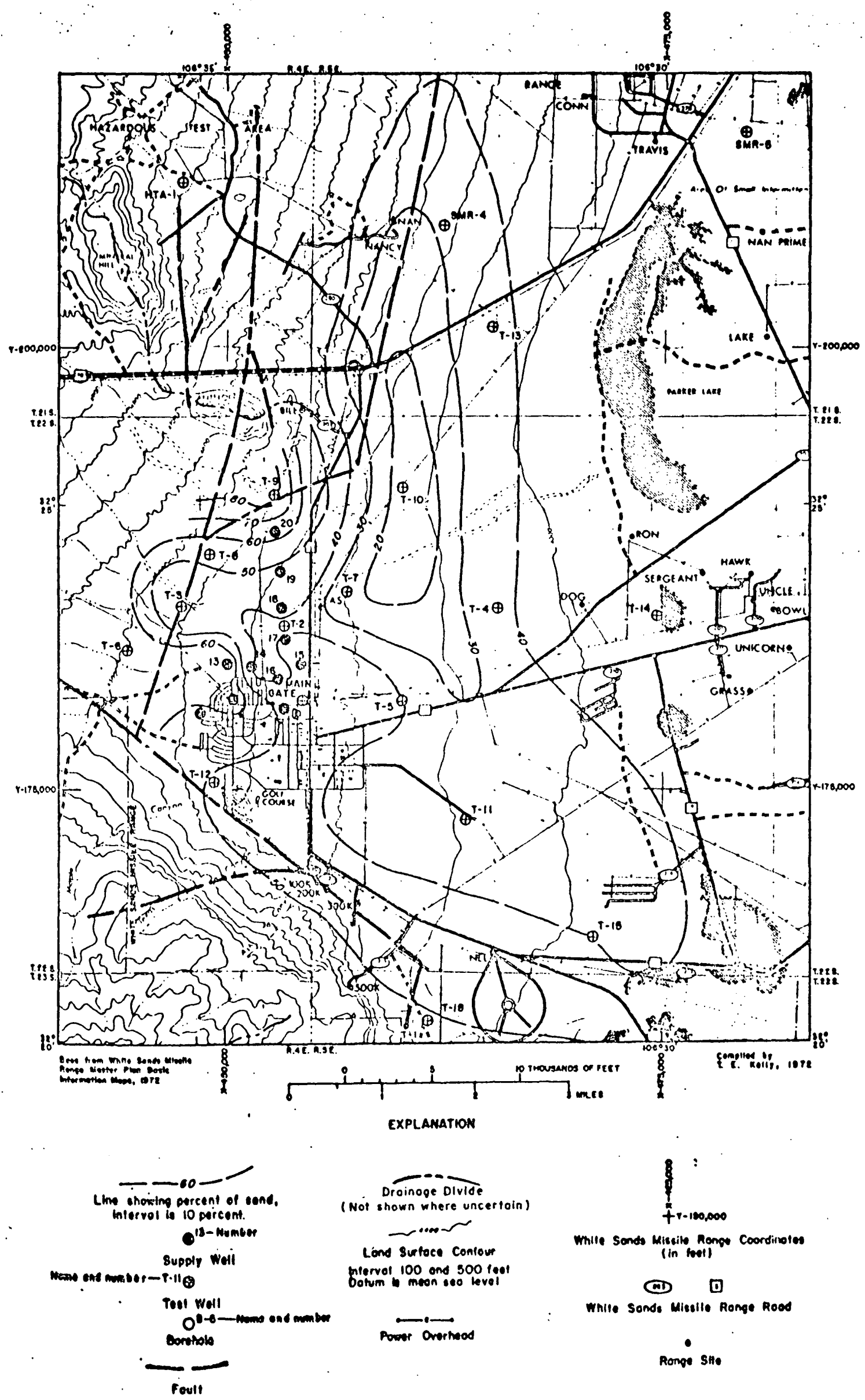

Figure 2.--Percentage of sand between 500 and 1,000 feet. 


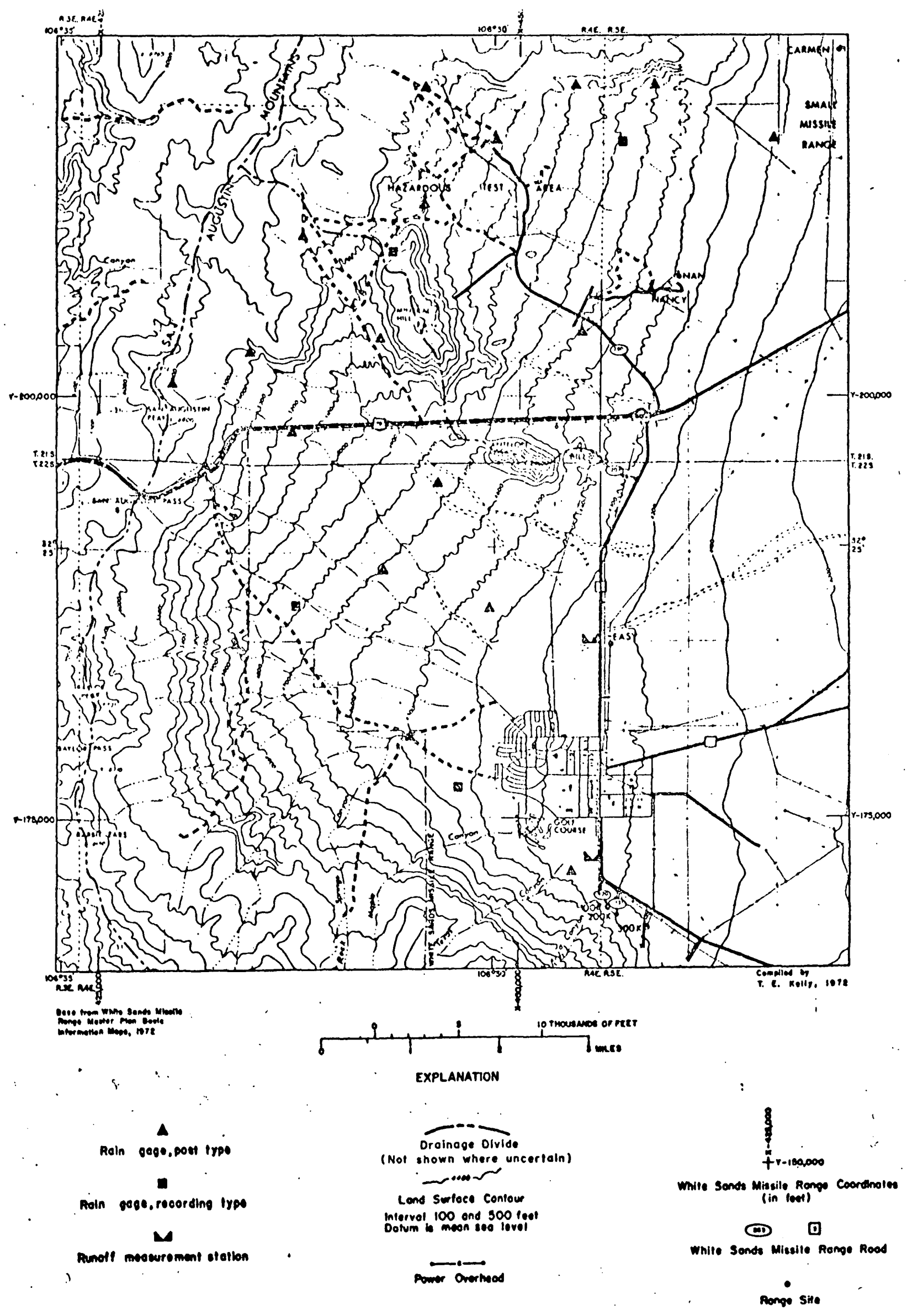

Figure 3.--Location of rain gages and surface-water stations. 
Accurate records of pumpage from wells in the Post Headquarters area have been kept by the Utilities Division, Facilities Engineering Directorate, since 1953 ( $f \mathrm{fg}$. 4). In general, there was a gradual increase in water use during the first 10 years of record. Subsequently the annual total production has ranged from 775.2 million gallons in 1966 to 939.0 million gallons in 1971; the average is 864.3 million gallons during the period 1964 to 1971 . The decline in water level recorded in Main Gate well (fig. 4) reflects the downward trend of the water table which has been caused by this withdrawal. 


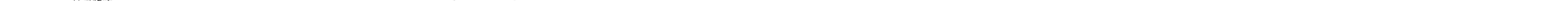


Figures 5 through 11 show the water-level changes that have occurred during the perfod of record in each test well. Most of these observation wells show a decline of water level; the amount of decline usually is dependent upon the location and proximity of the observation well to the well field.

The annual pumpage and corresponding water-level changes for each of the currently operative supply wells are illustrated in figures 12 through 20. Most of these wells have declining water levels. However since about 1965, wells 15 through 20 have been the major source of supply, thus the water levels have risen in supply wells 11 and 13 (figs. 13 and 14).

Water levels have been measured intermittently in supply wells HTA-1 and SMR-1 (fig. 21). These wells produce small quantities of water for local use. Two wells, 12 and 14 , have been utilized as observation wells after having been abandoned as supply wells (fig. 22). Supply well 12 reflects the rise in water table near the south end of the well field.

W. C. Ballance and S. M. Longwill (written commun., 1968) analyzed much of the data that was collected prior to 1964 . This early work provided a background for construction of various maps illustrating the early well development in the Post Headquarters area. 


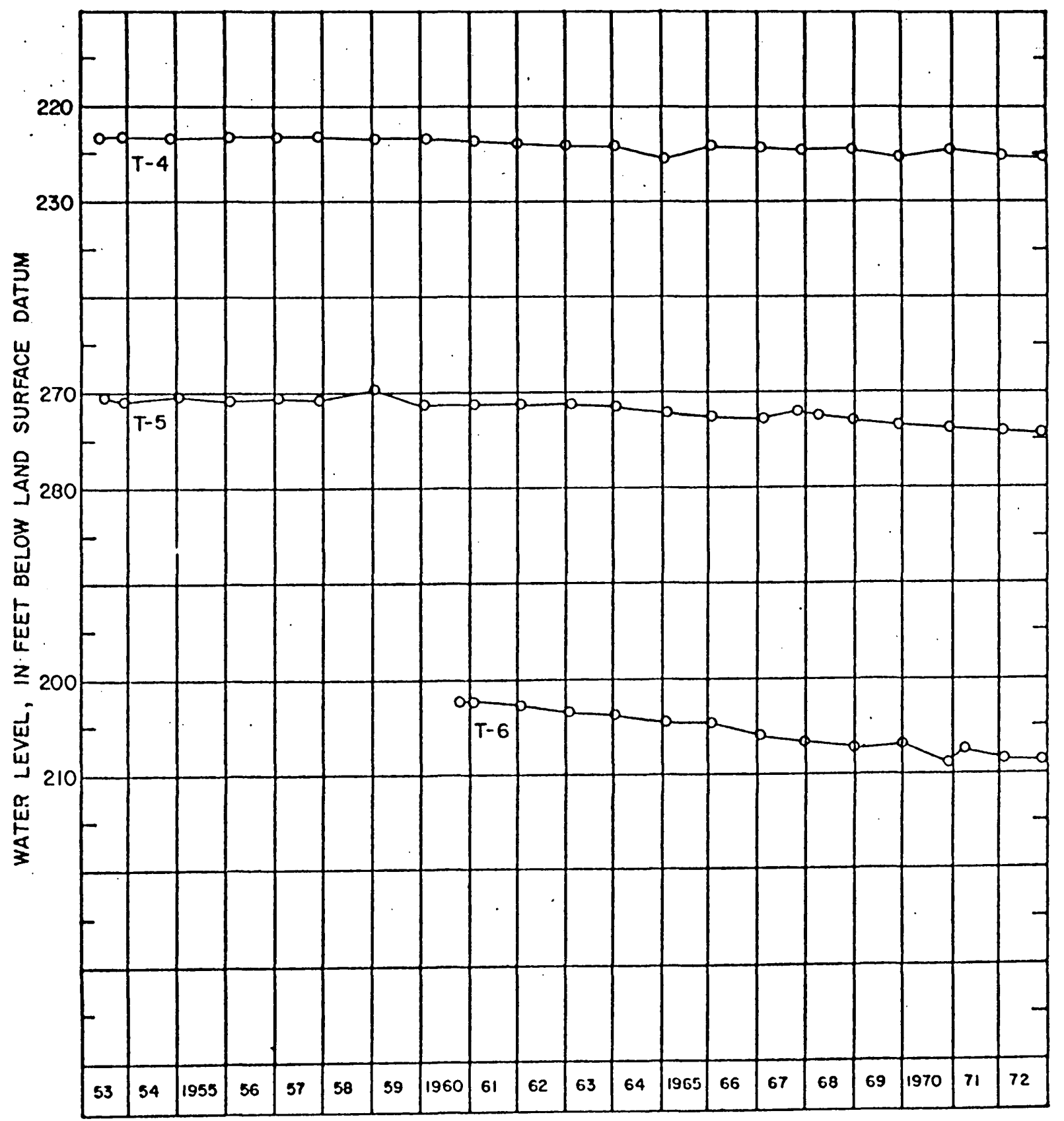

Figure 5.--Hydrograph of Test wells $T-4, T-5$, and $T-6$. 


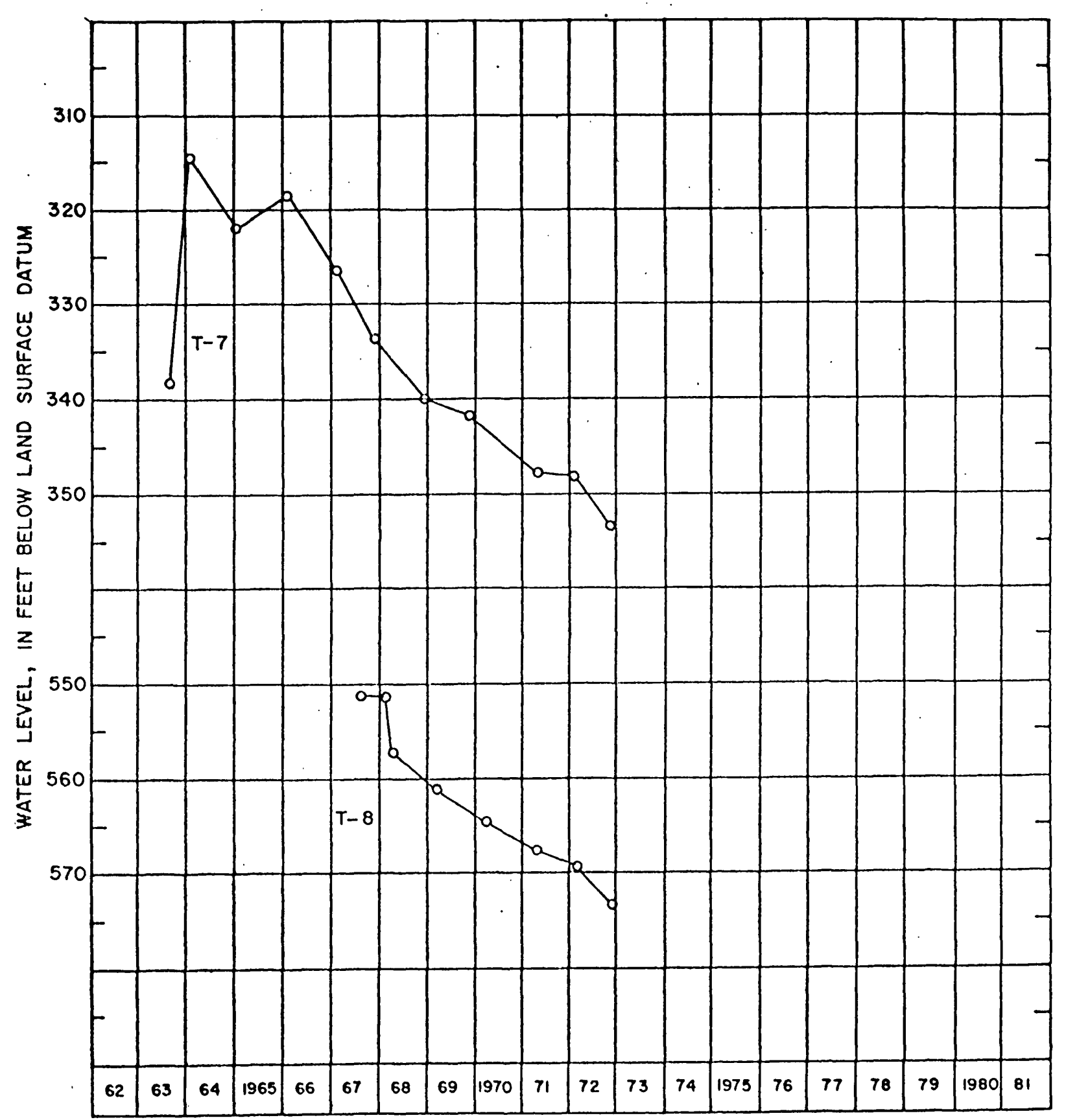

Figure 6.--Hydrograph of Test wells T-7 and T-8. 


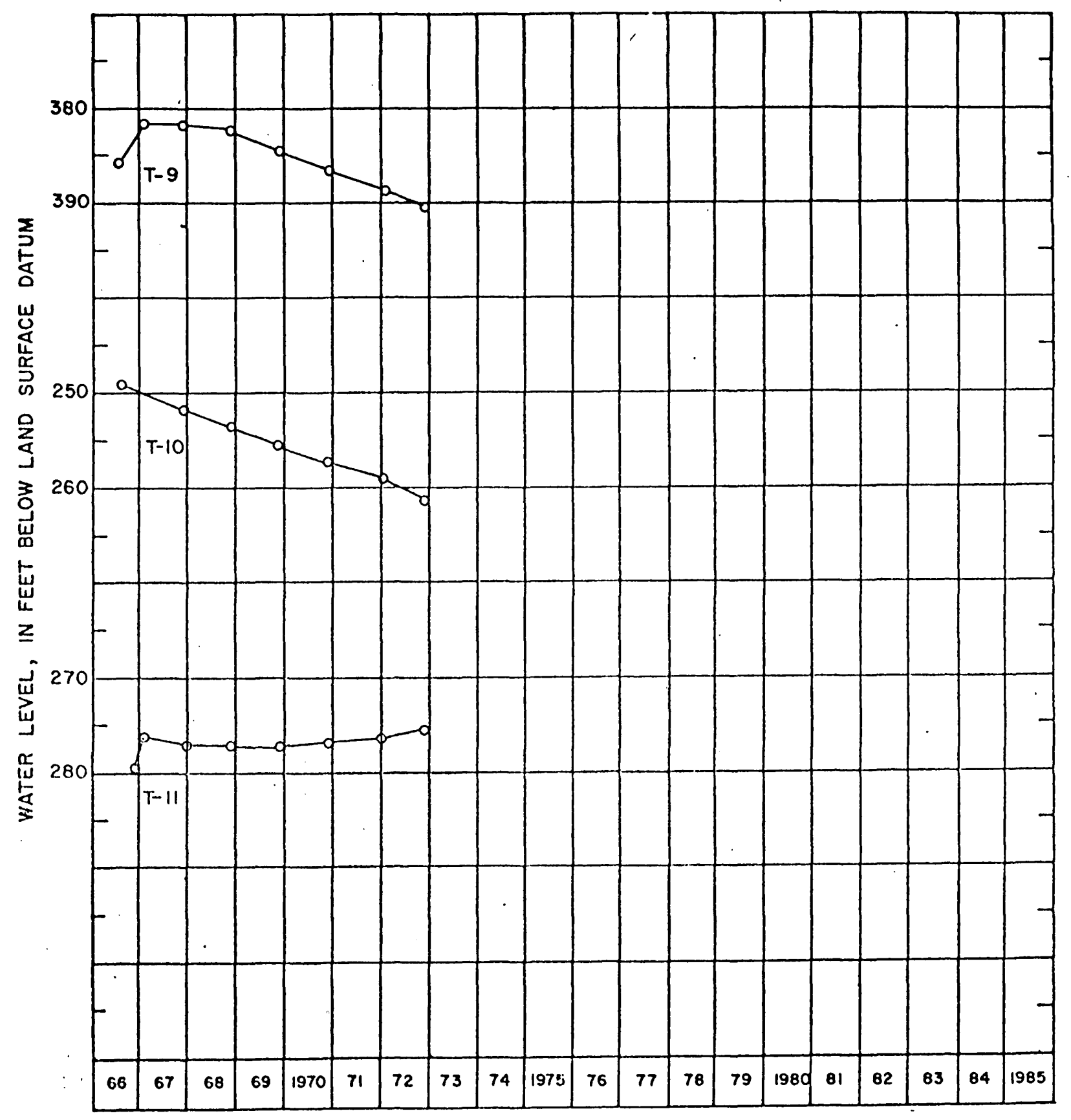

Figure 7.--Hydrograph of Test wells $T-9, T-10$, and $T-11$. 


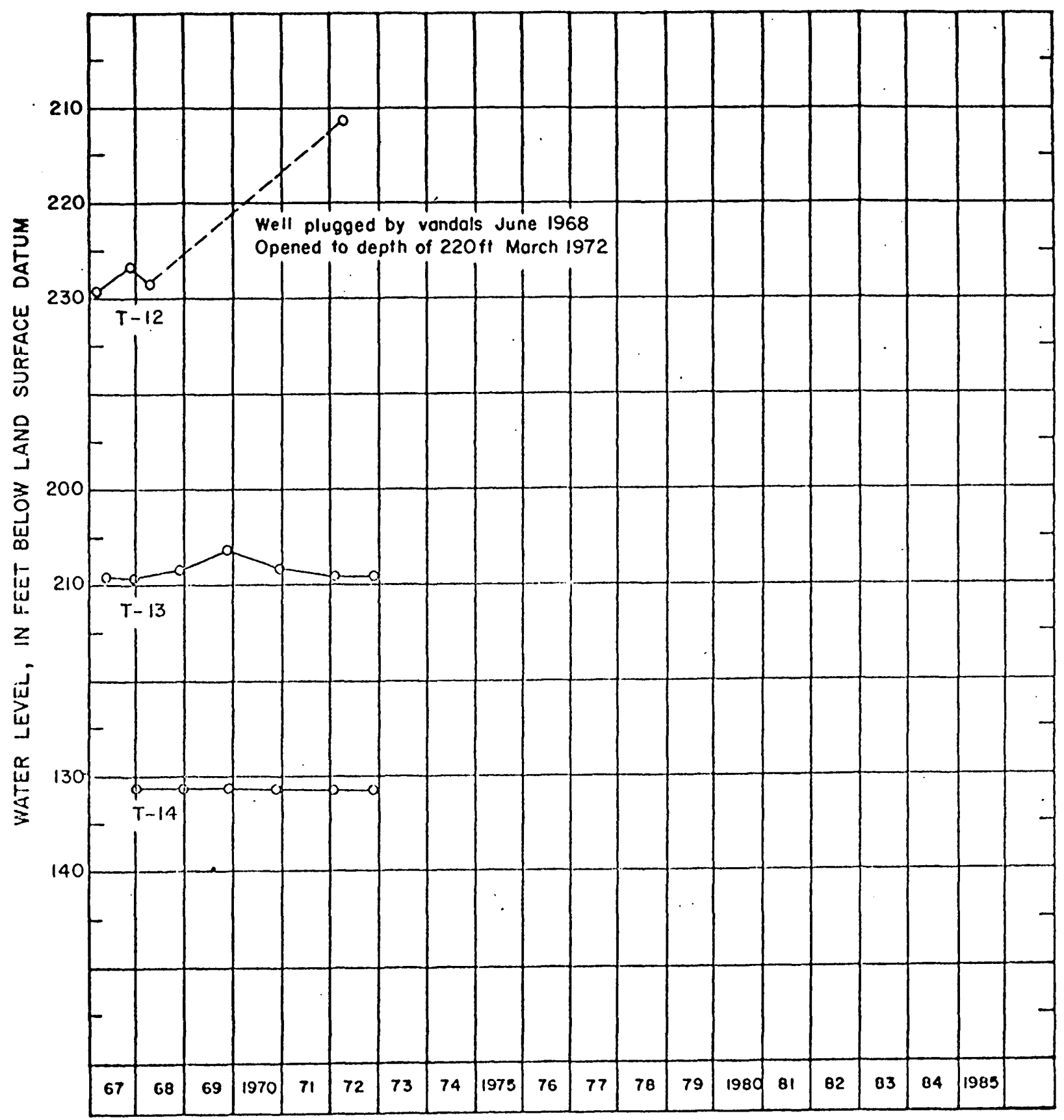

Figure 8.--Hydrograph of Test wells $T-12, T-13$, and $T-14$. 


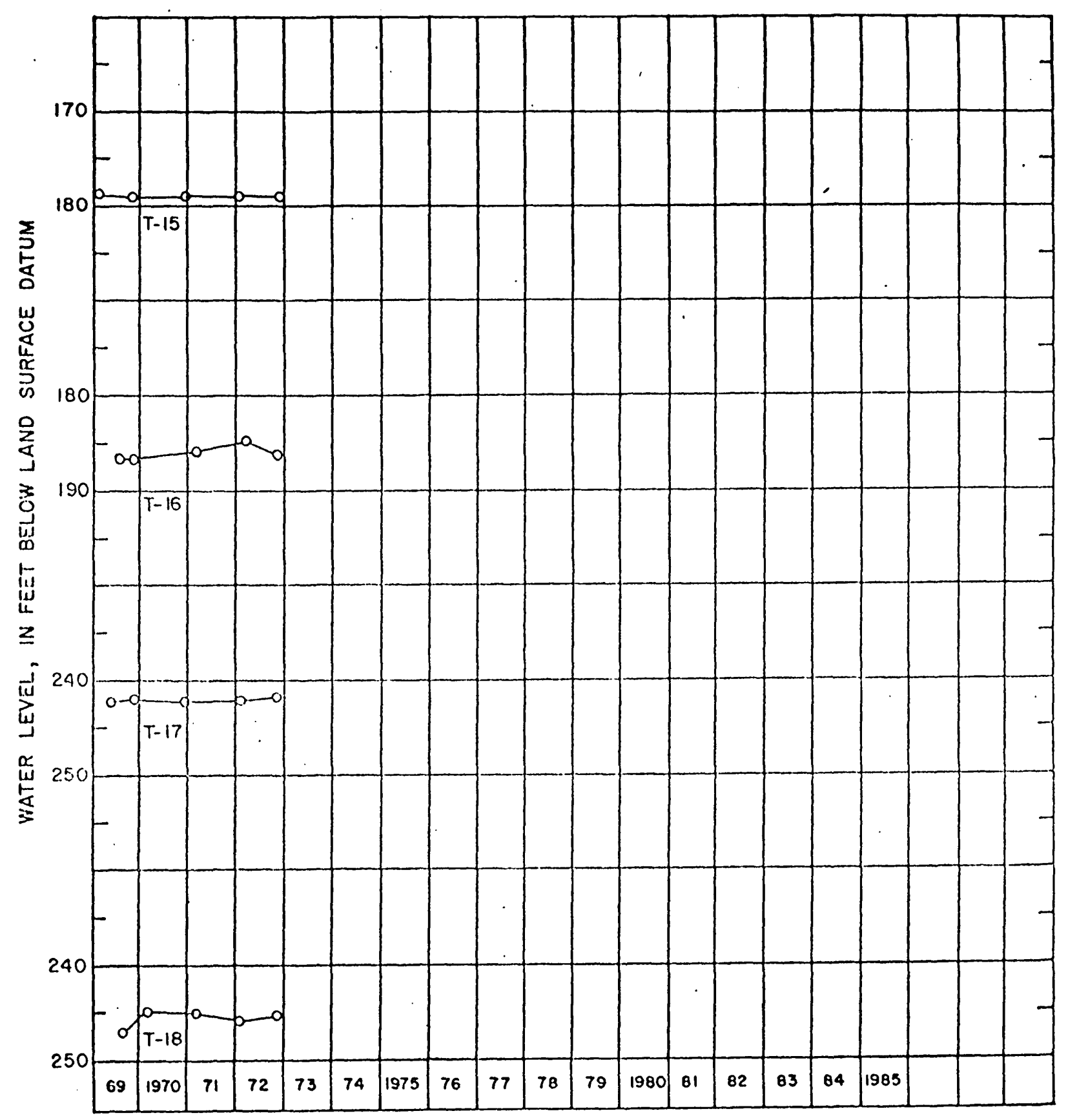

Figure 9.--Hydrograph of Test wells T-15,T-16, T-17, and T-18. 


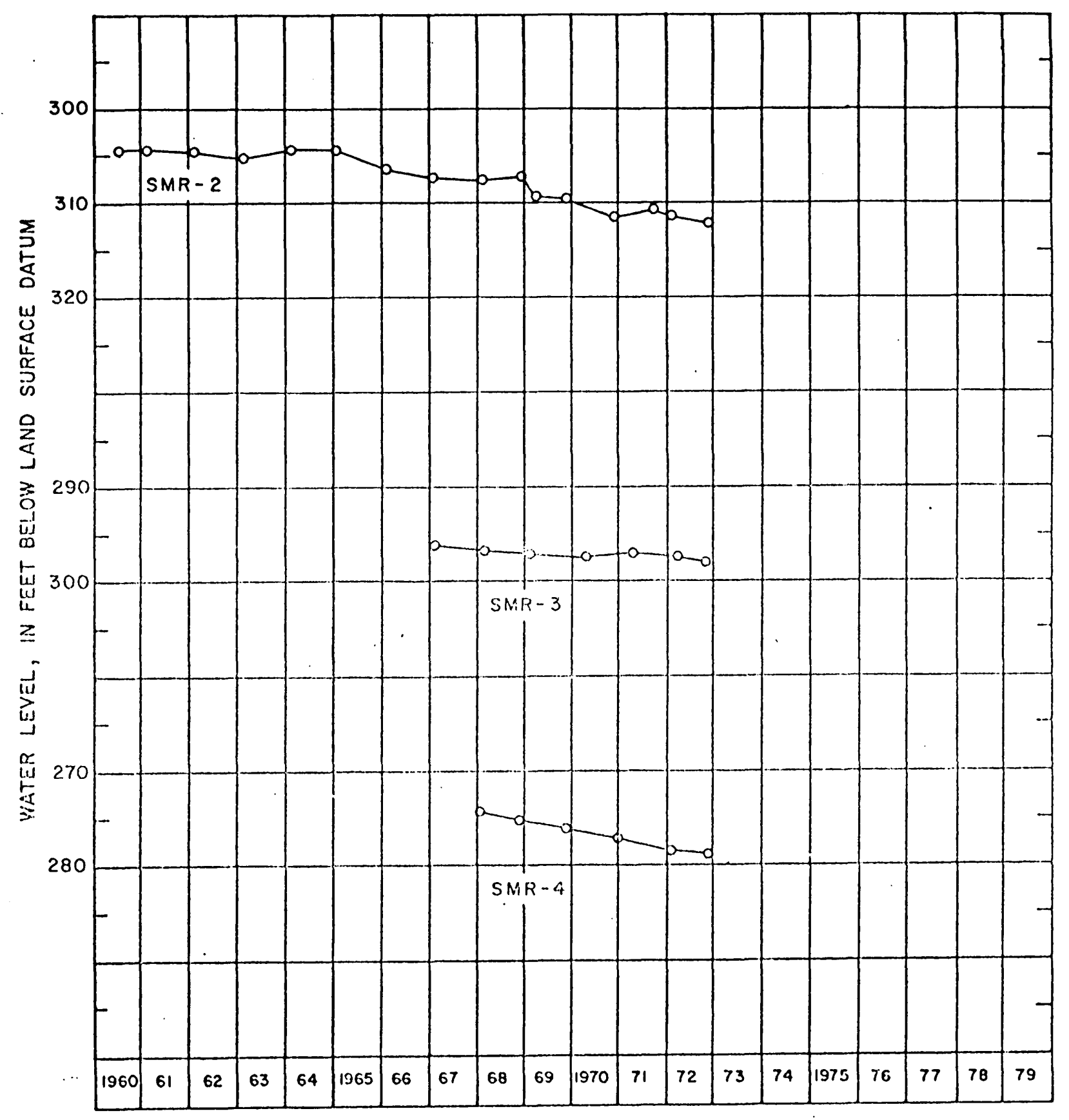

Figure 10.--Hydrograph of Test wells SMR-2, SMR-3, and SMR-4. 


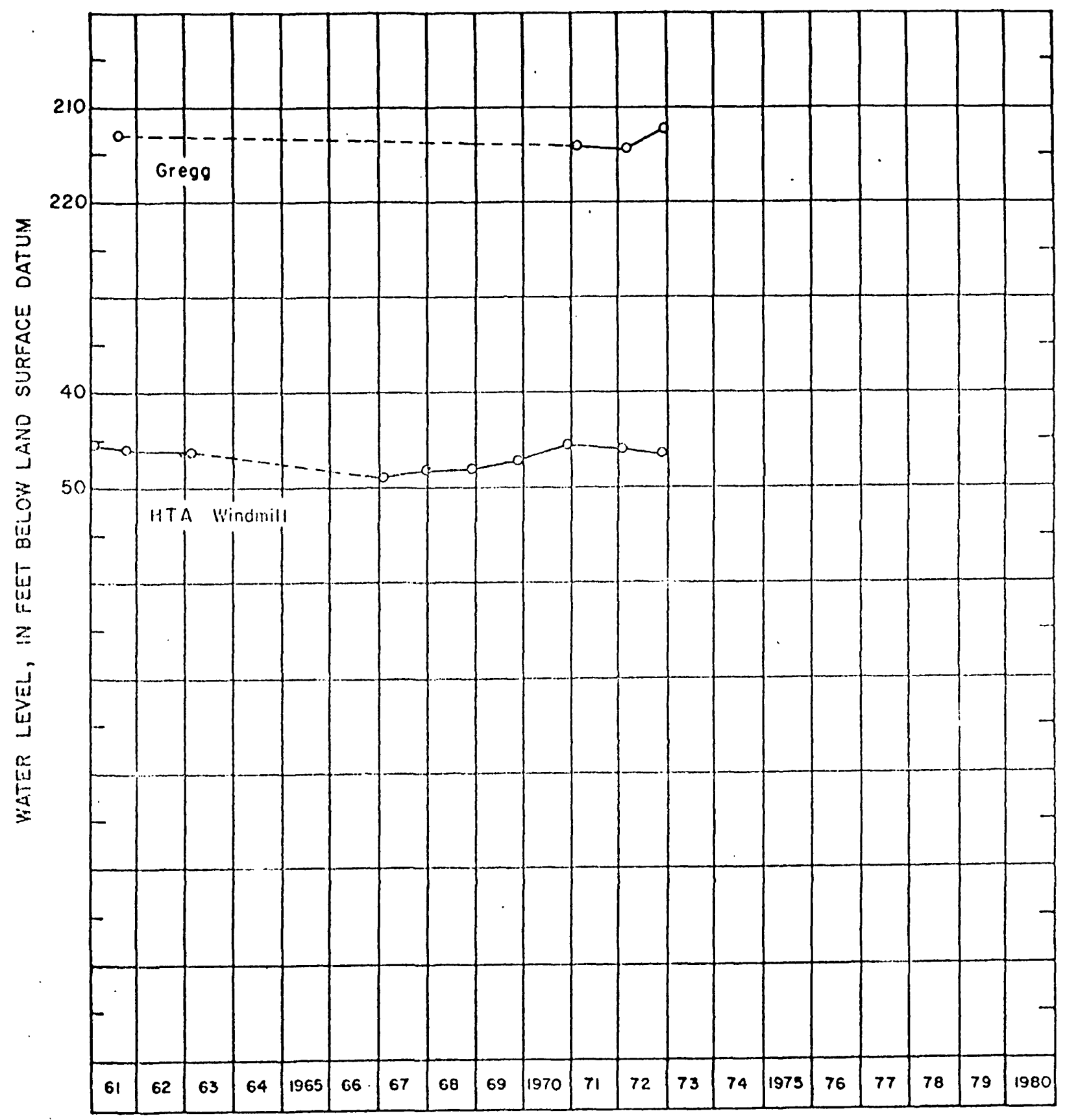

Figure 11 .--Hydrograph of Gregg well and.HTA windmill. 


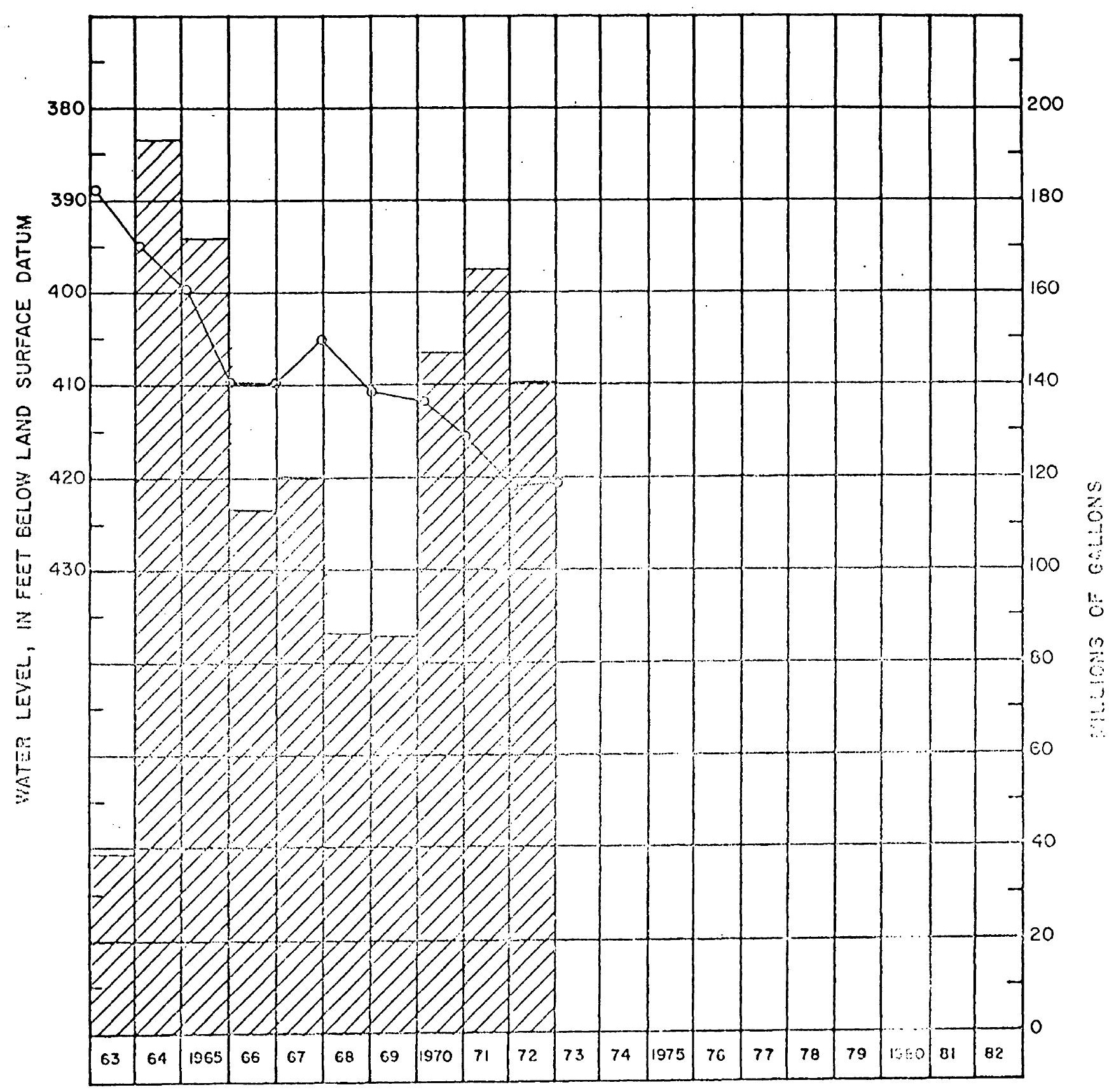

Figure 12.--Annual pumpage and hydrograph of supply well 10A. 


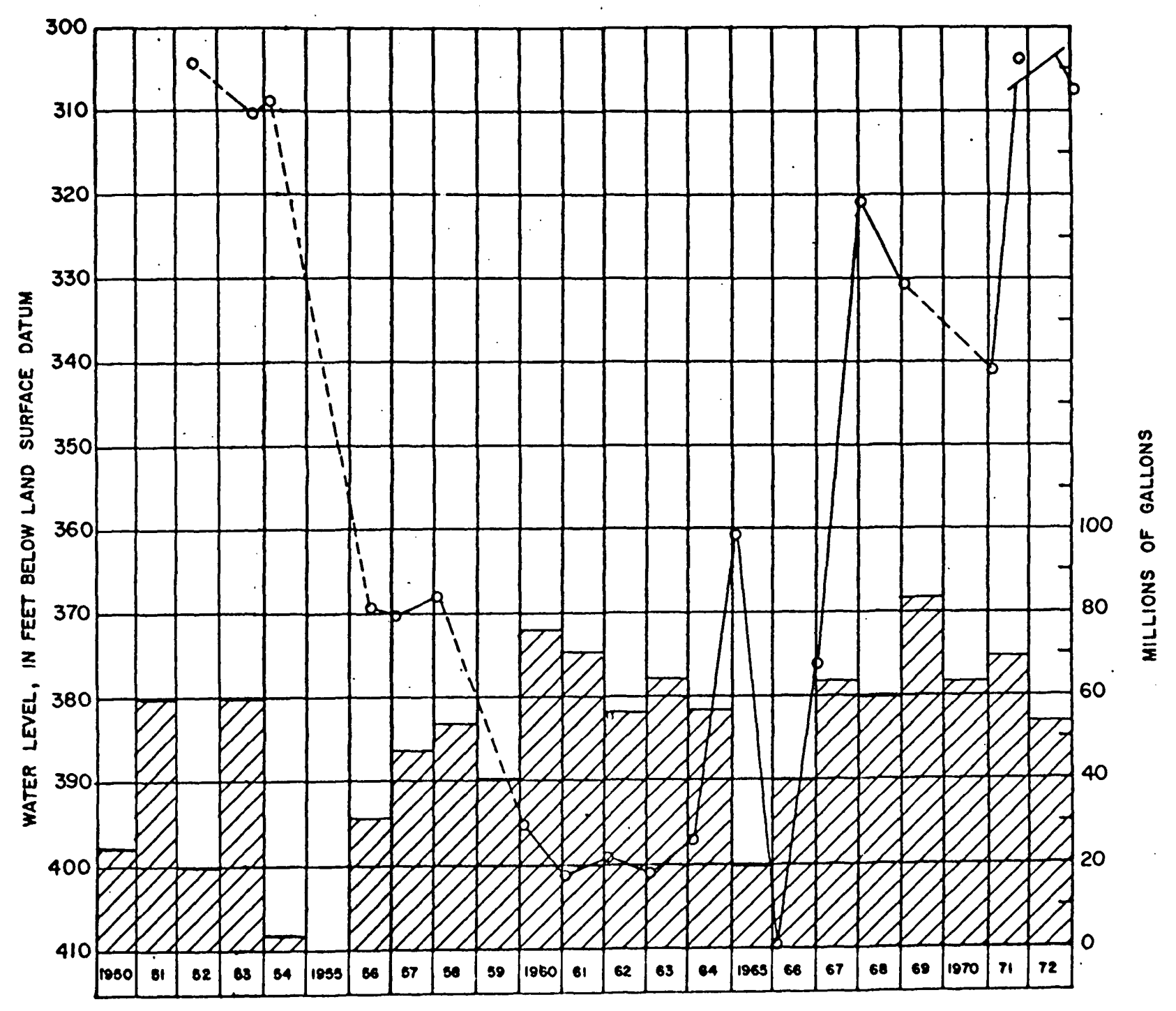

Figure 13.--Annual pumpage and hydrograph of supply well 11. 


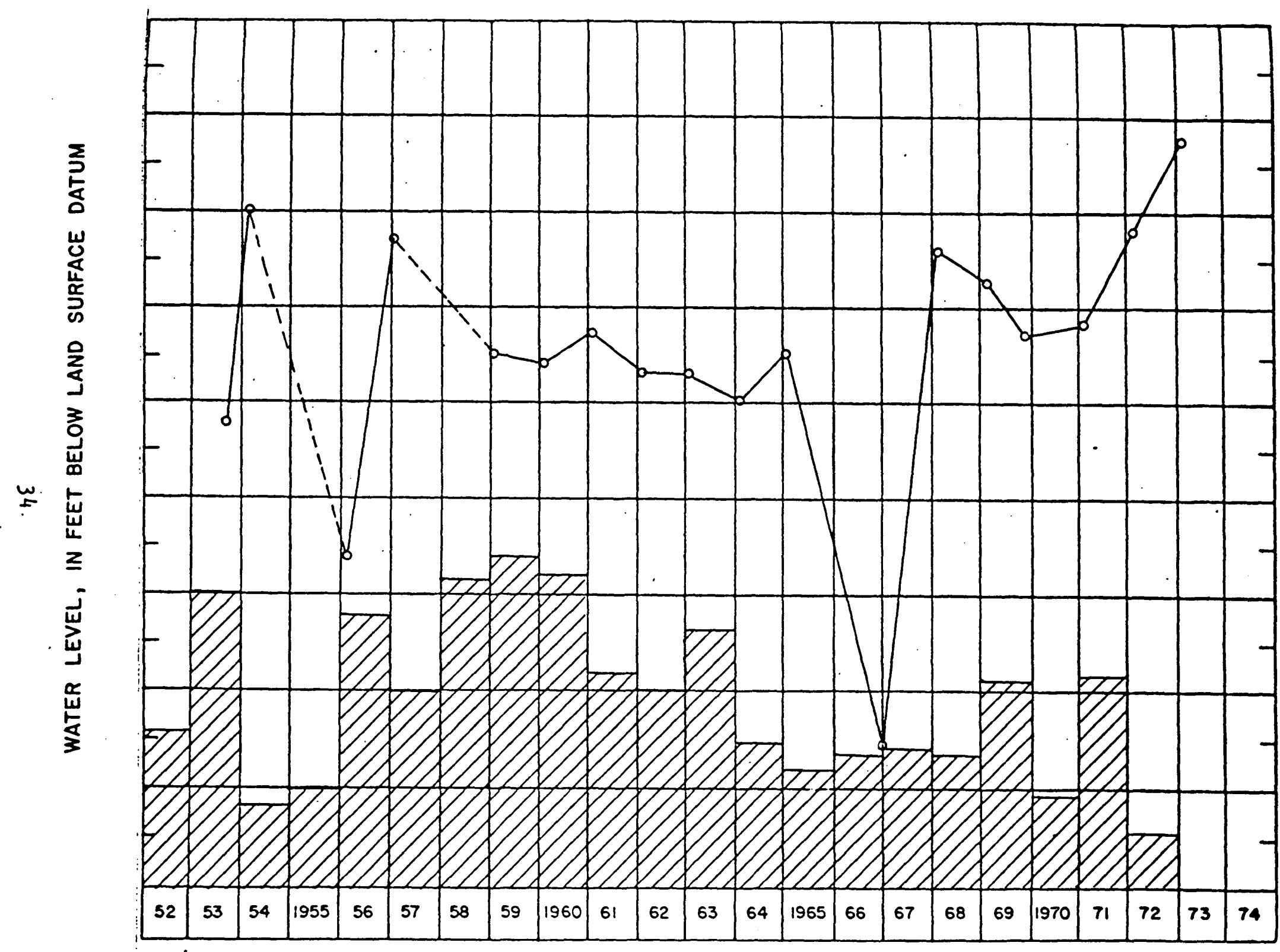

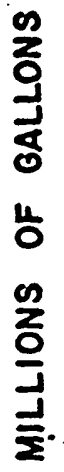

Figure 14.--Annual pumpage and hydrograph of supply well 13. 


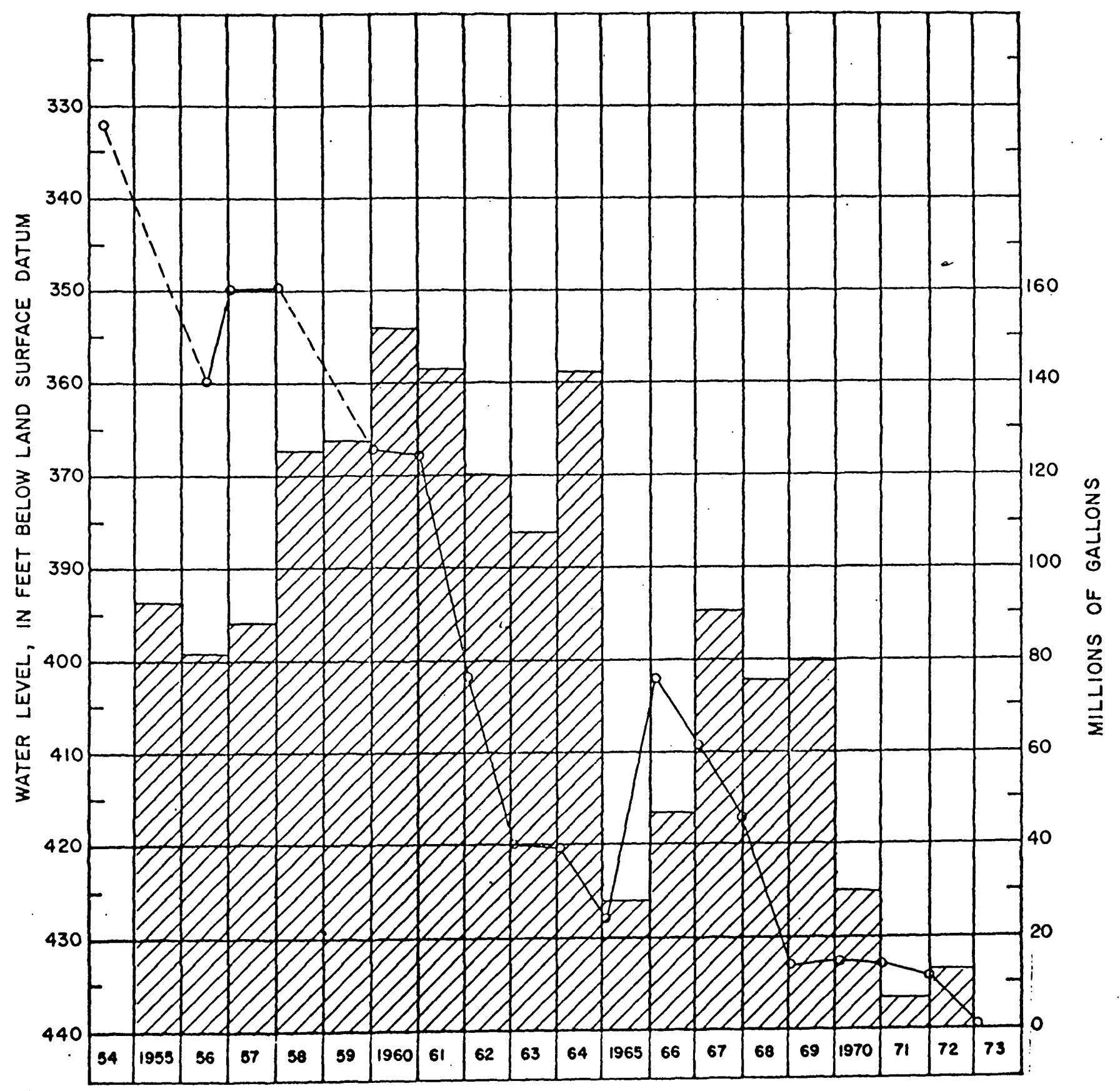

Figure 15.--Annual pumpage and hydrograph of supply well 15. 


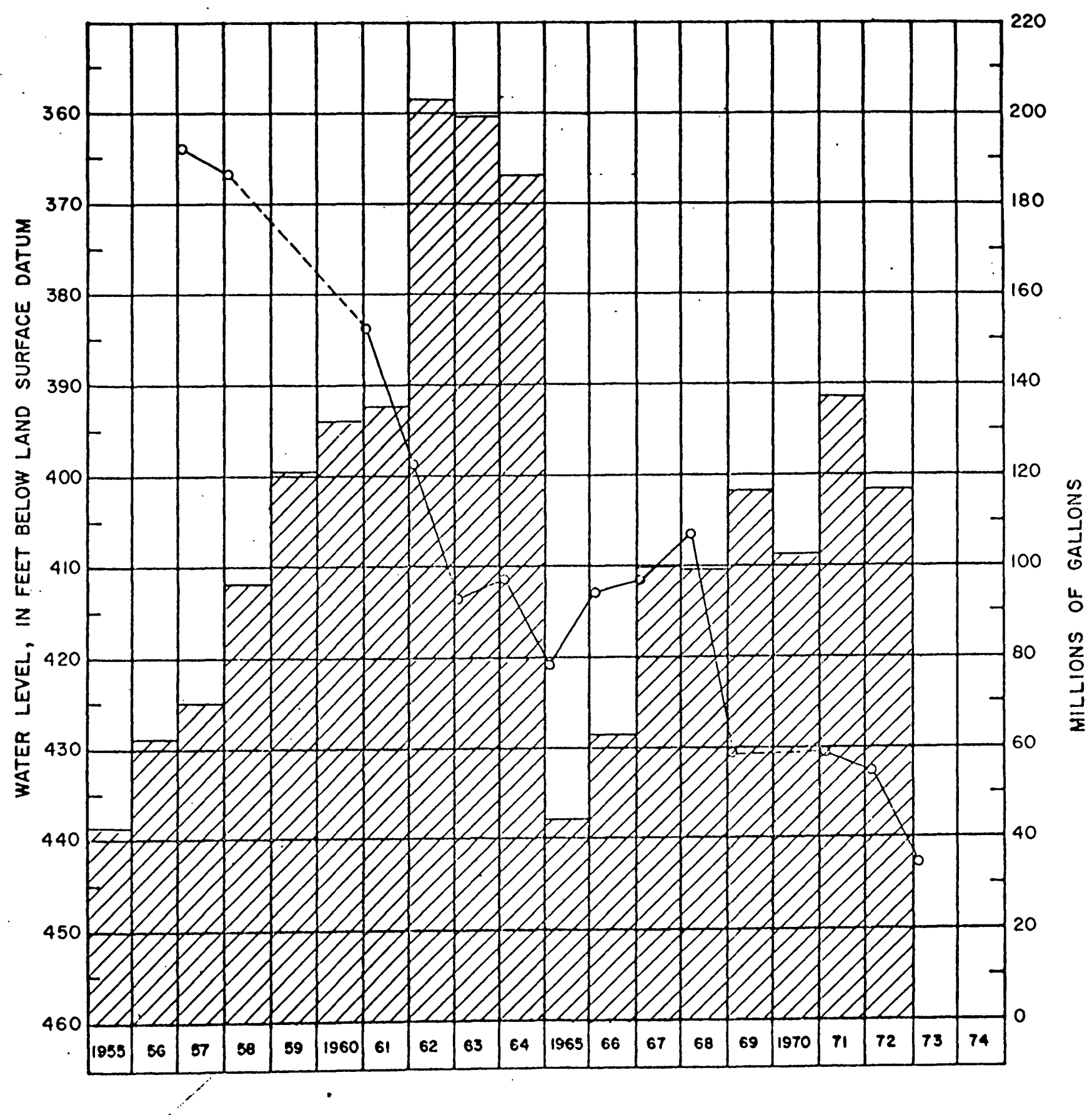

Figure 16.--Annual pumpage and hydrograph of supply well 16. 


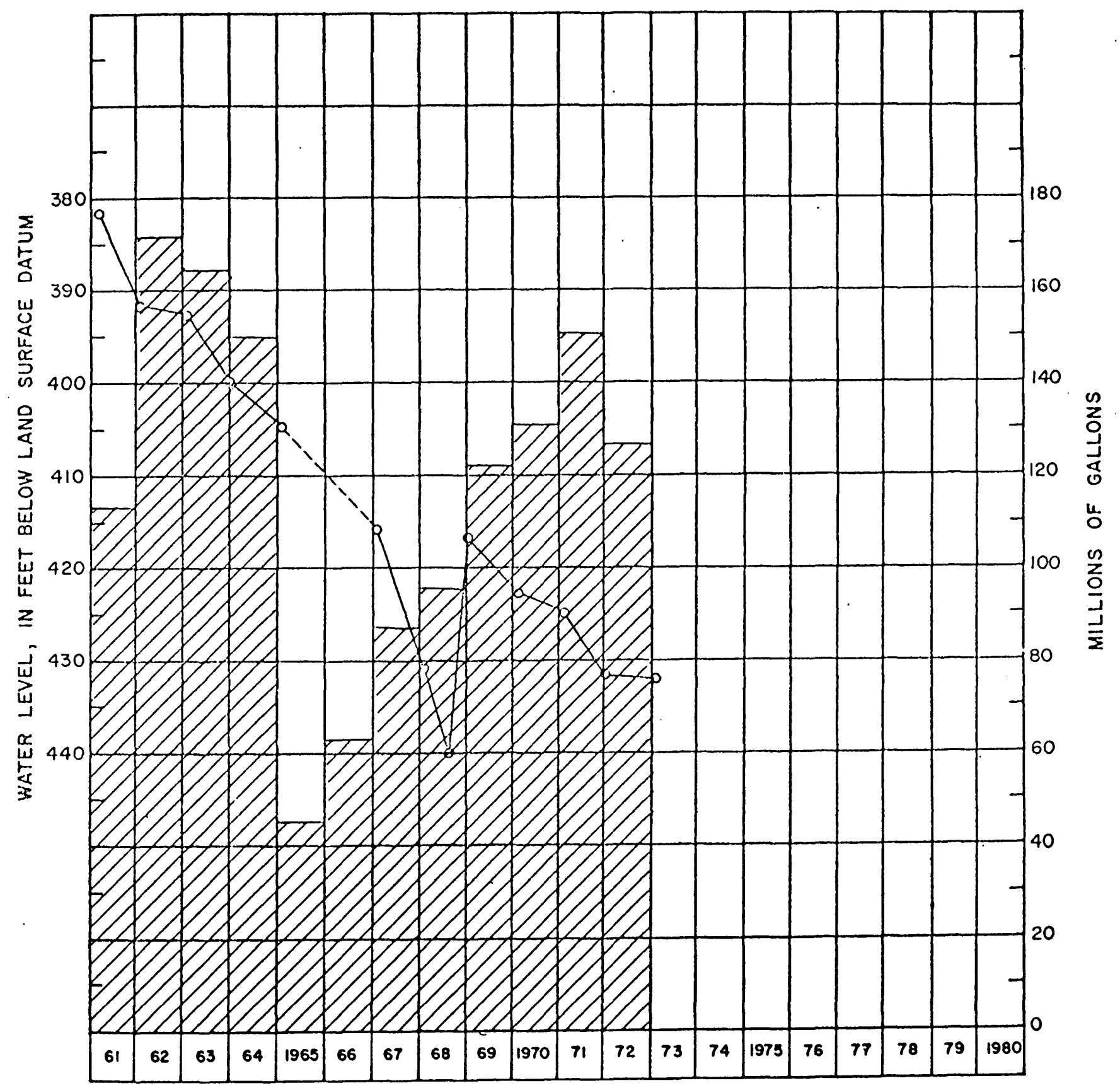

Figure 17.--Annual pumpage and hydrograph of supply well 17. 


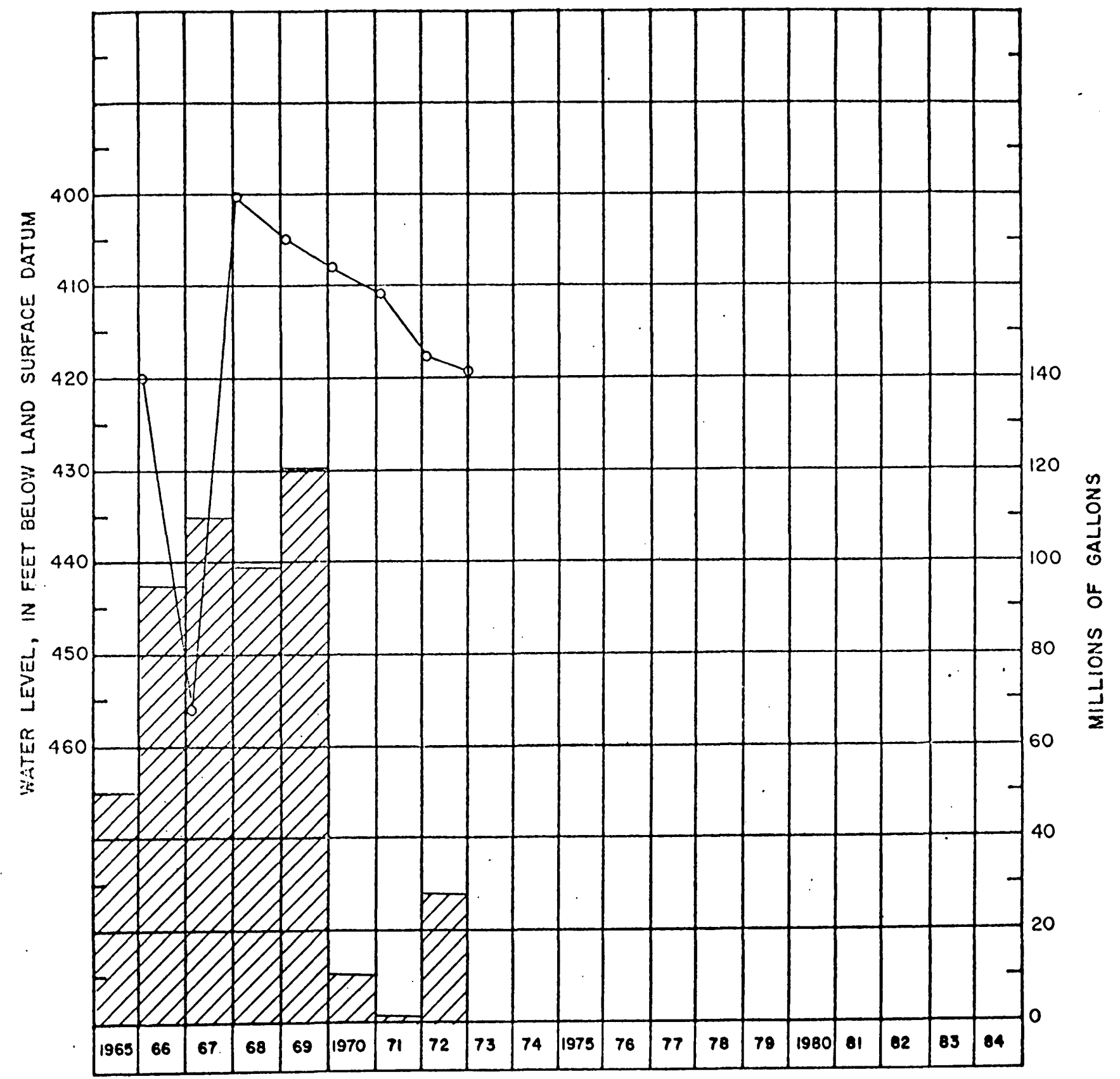

Figure 18.--Annual pumpage and hydrograph of supply well 18. 


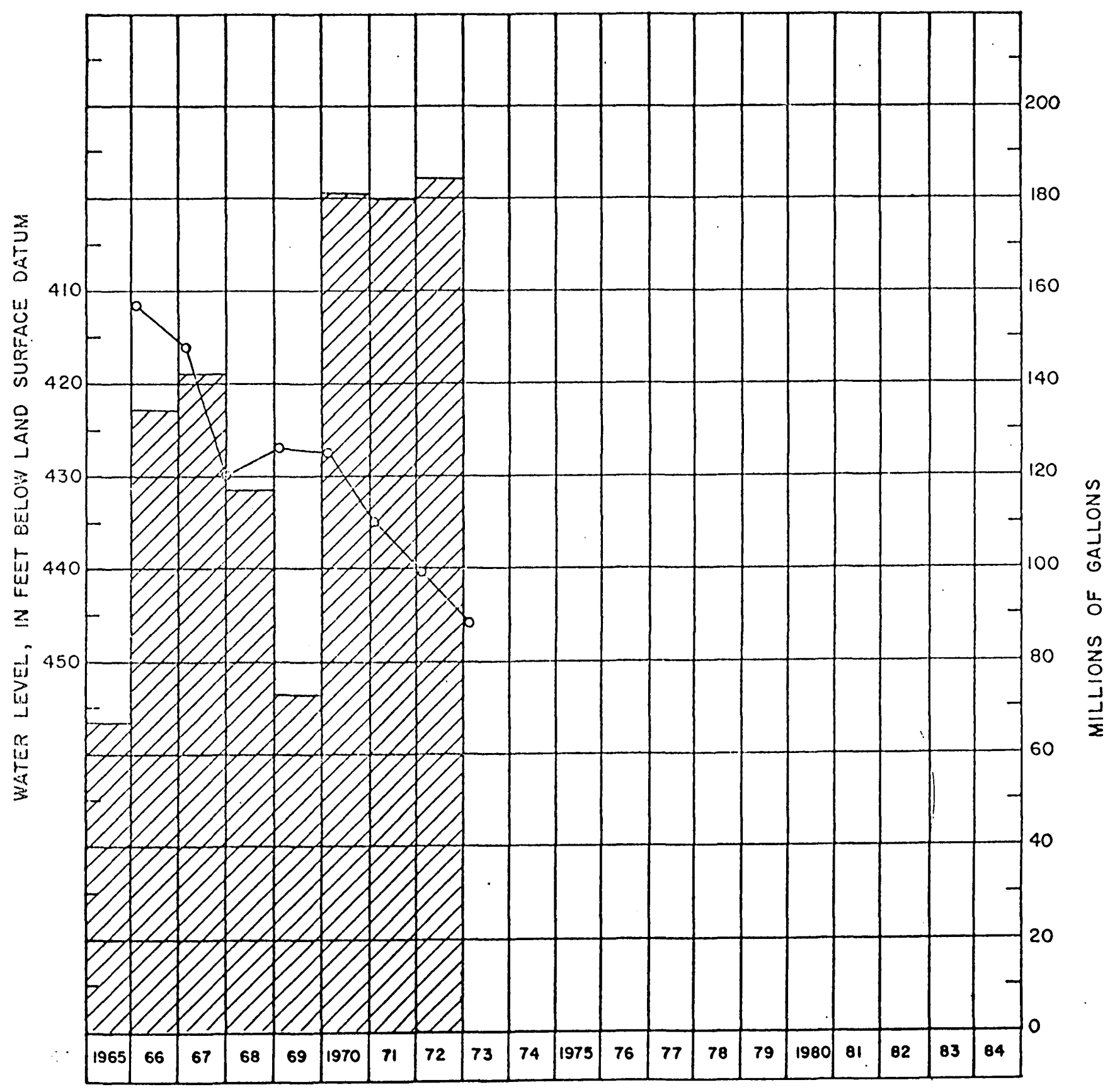

Figure 19.--Annual pumpage and hydrograph of supply well 19. 


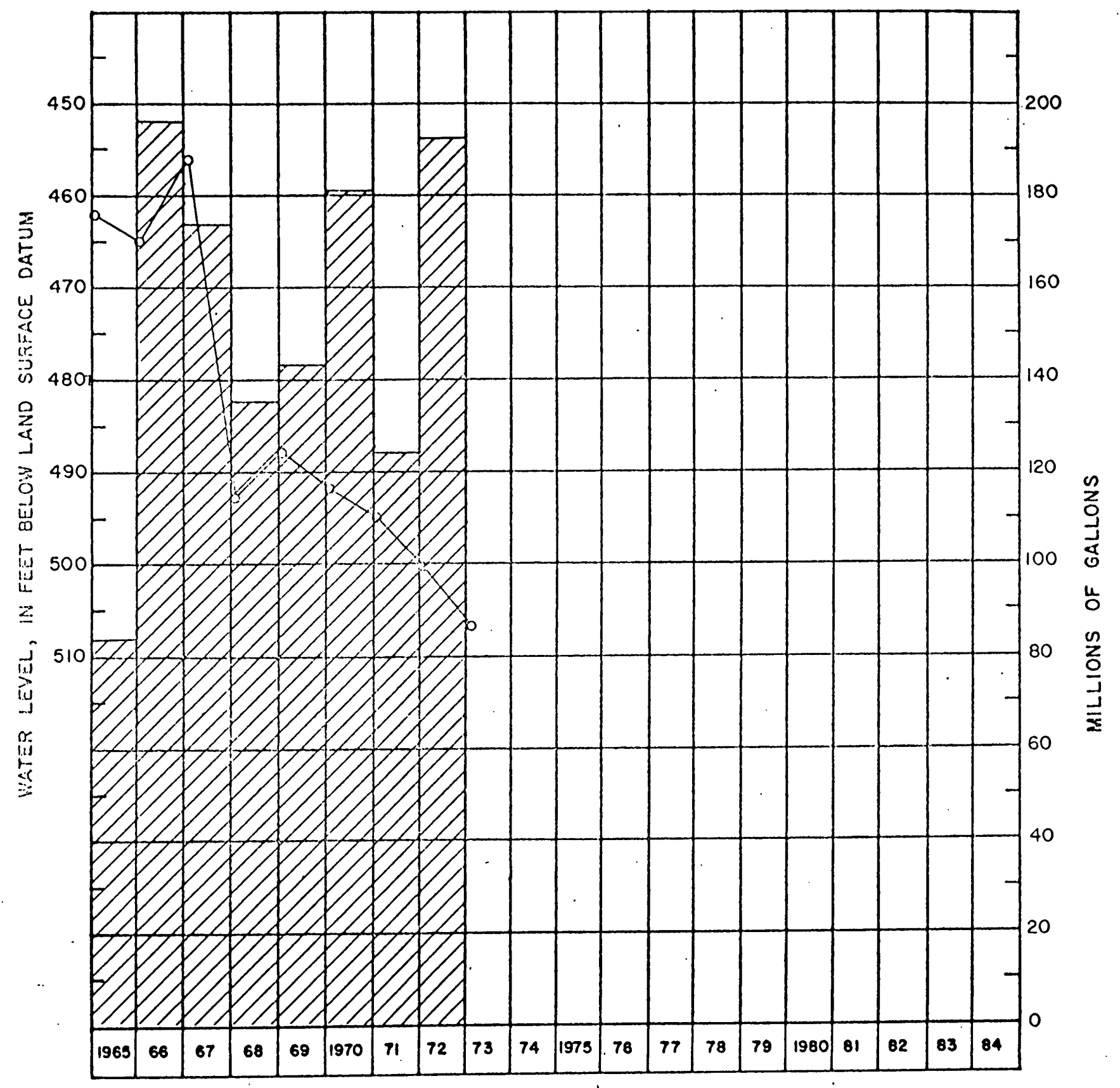

Figure 20.--Annual pumpage and hydrograph of supply well 20. 


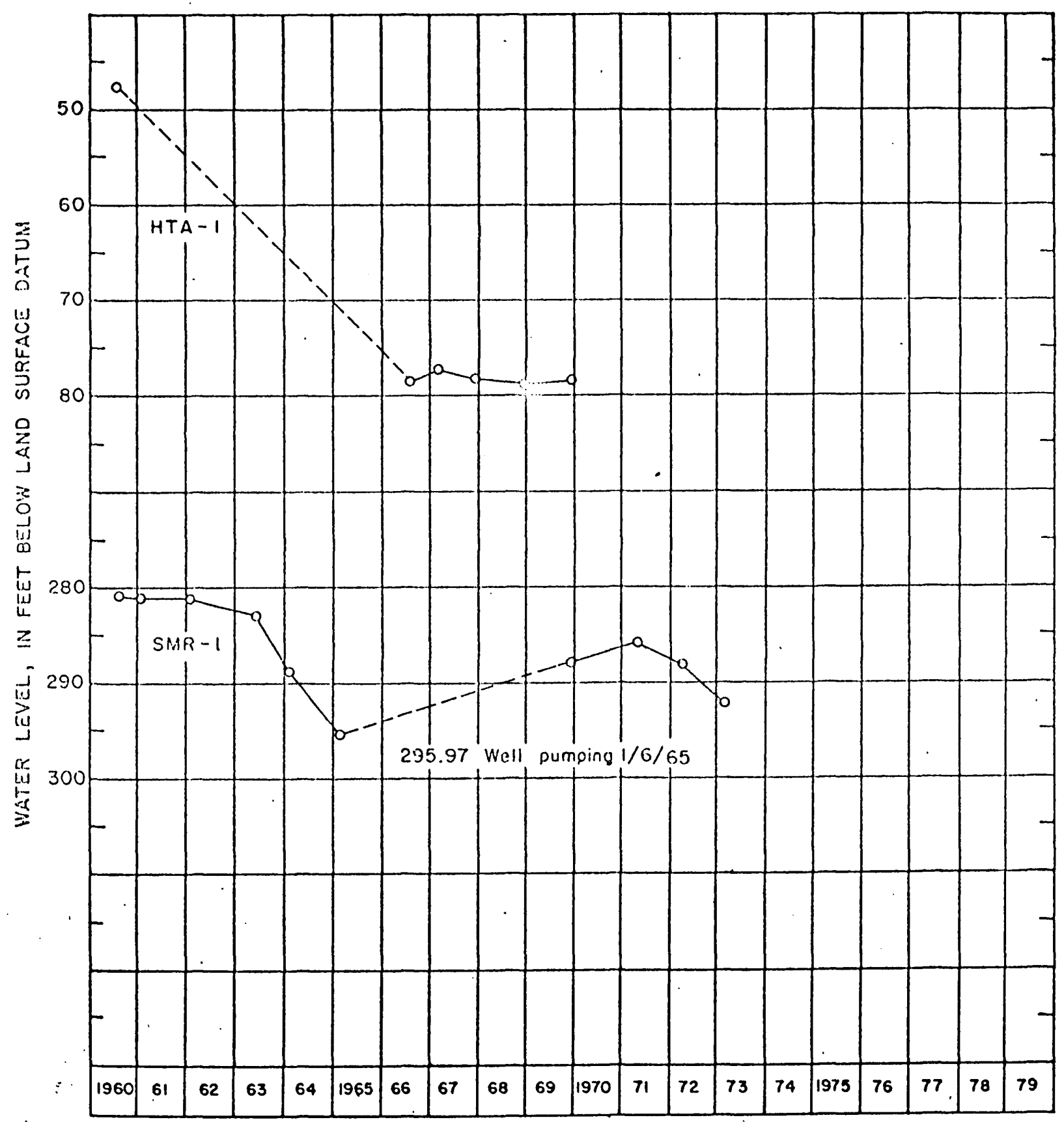

Figure 21.--Hydrograph of supply wells HTA-1 and SMR-1. 


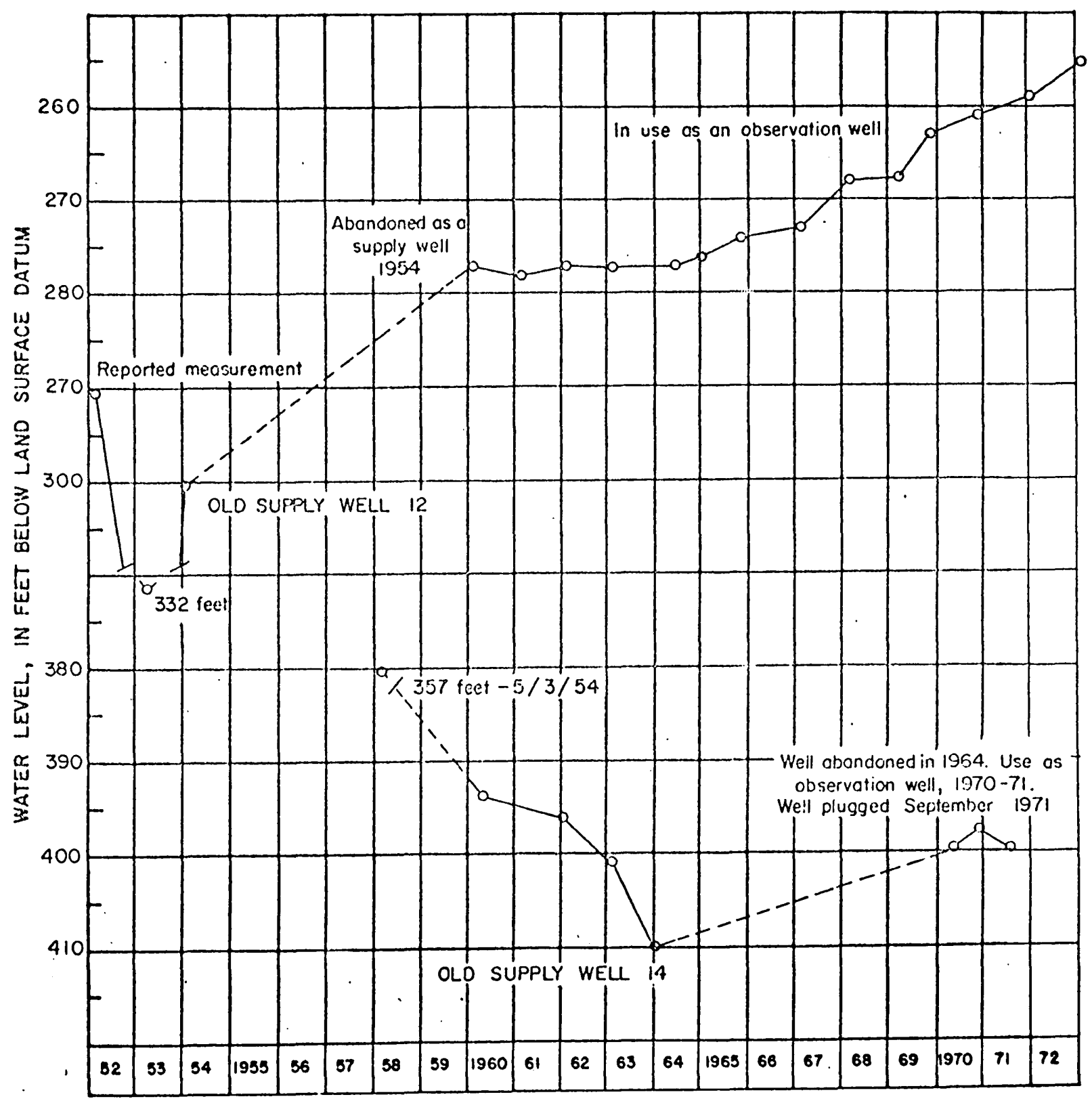

Figure 22.--Hydrograph of old supply wells 12 and 14: 
The estimated configuration of the water table in 1949 is shown on figure 23. Although only a few stock wells provided the control, this map shows a regional slope of the water table from the nearby mountains to the Tularosa Basin across the White Sands reentrant. Undoubtedly this reflects the ground-water conditions prior to development.

After 15 years of production from the Post Headquarters well fleld, a clearly defined cone of depression existed (fig. 24). Although there had been little change in the water-table contours west of the well field, the contours east of the field showed a pronounced influence due to pumping.

During the period 1949 through 1964, an estimated 20,000 acrefeet of water was withdrawn from the aquifer beneath the Post Headquarters well field. The maximum drawdown was slightly more than 75 feet in the area of maximum production (fig. 25). At that time (1964) the cone of depression had not extended beyond the rcentrant. In July 1972, the axis of the cone of depression roughly paralleled the line of supply wells (fig. 26). The area influcnced by pumping had extended to the limits of the reentrant, and eastward into the Tularosa Basin.

From 1964 until July 1972, approximately 20,000 acre-feet of water was withdrawn from the aquifer. This caused an additional drawdown of more than 25 feet in the four major producing wells (fig. 27). Unlike the drawdown shown in figure 25 for the 1949-1964 interval, the area of influence was spread more evenly throughout the well field. Also, wells 12 and 14 were not in production during this time, so there was a general recovery in the southwest part of the well field. 


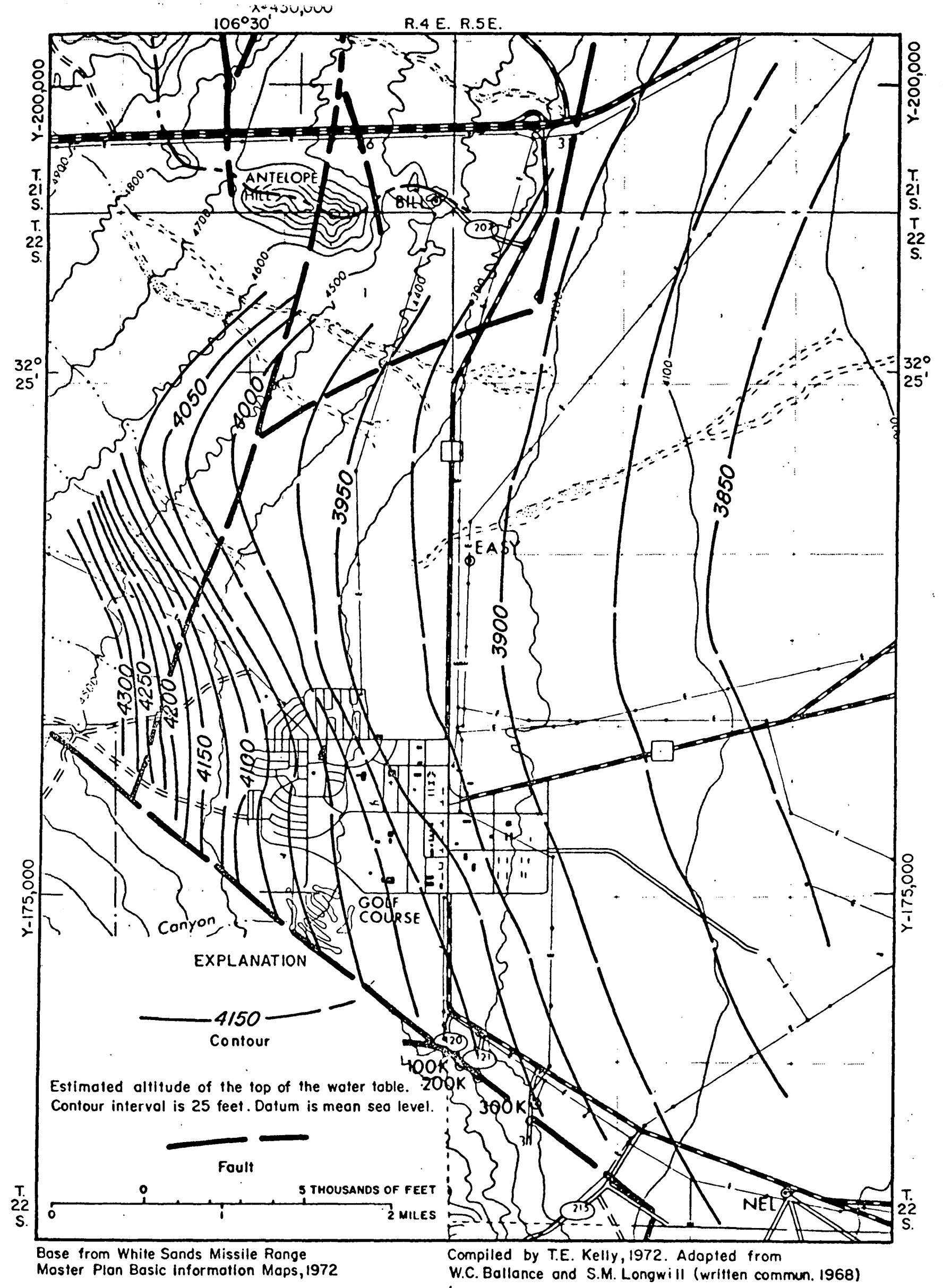

Figure 23.-- Contour map of water table, 1949 (estimated). 


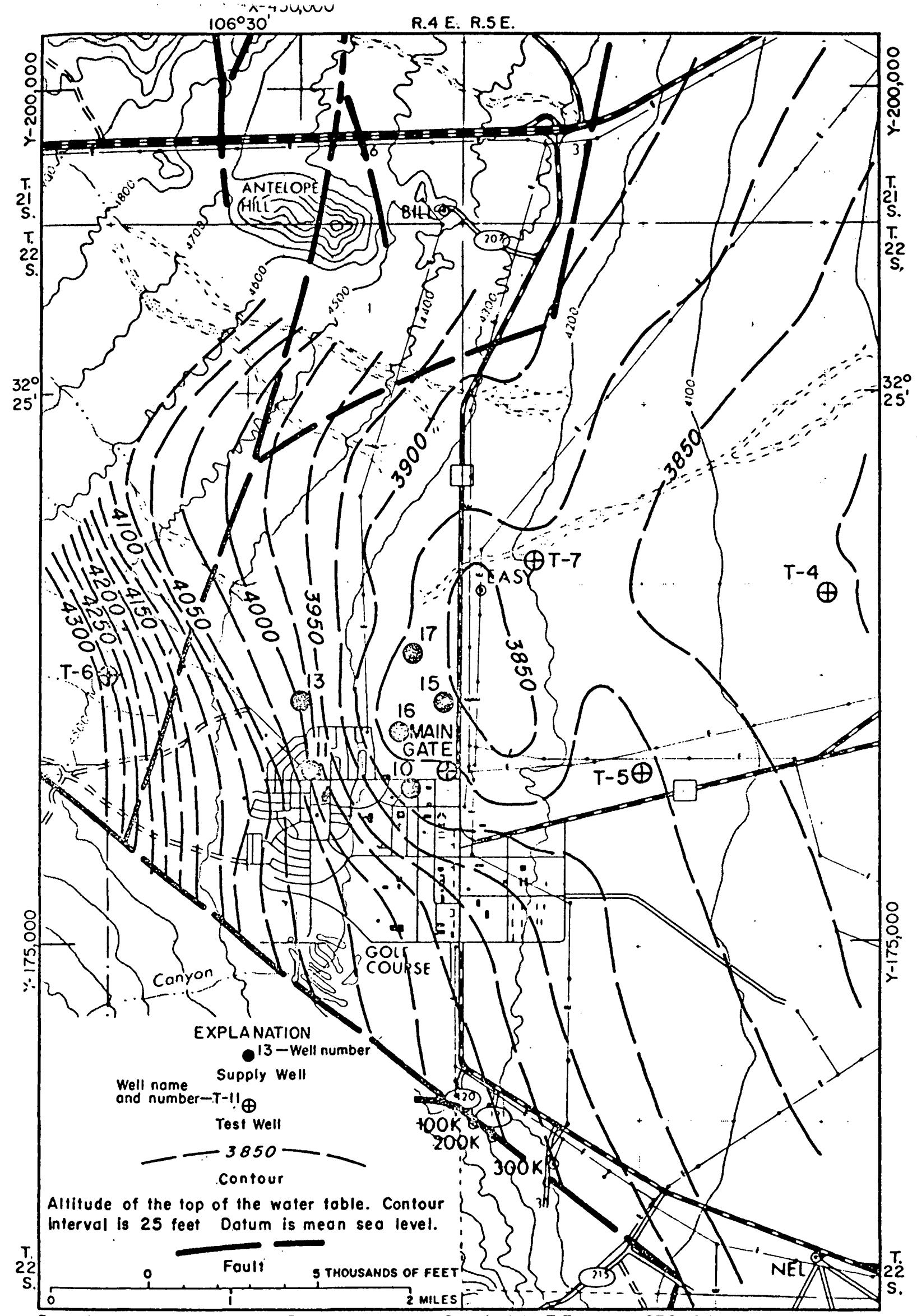




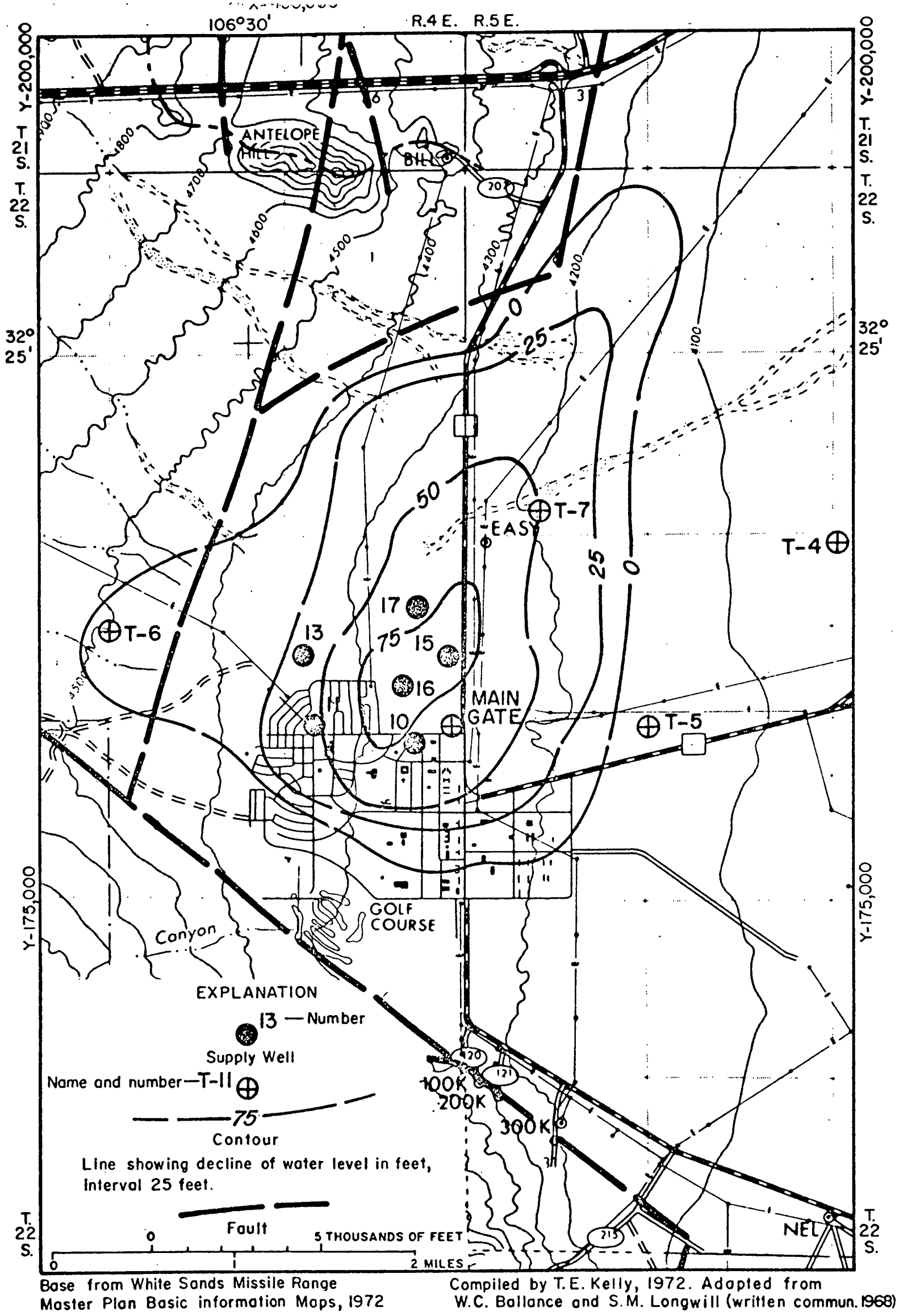

Figure 25.--Water-level change between 1949 and 1964. 


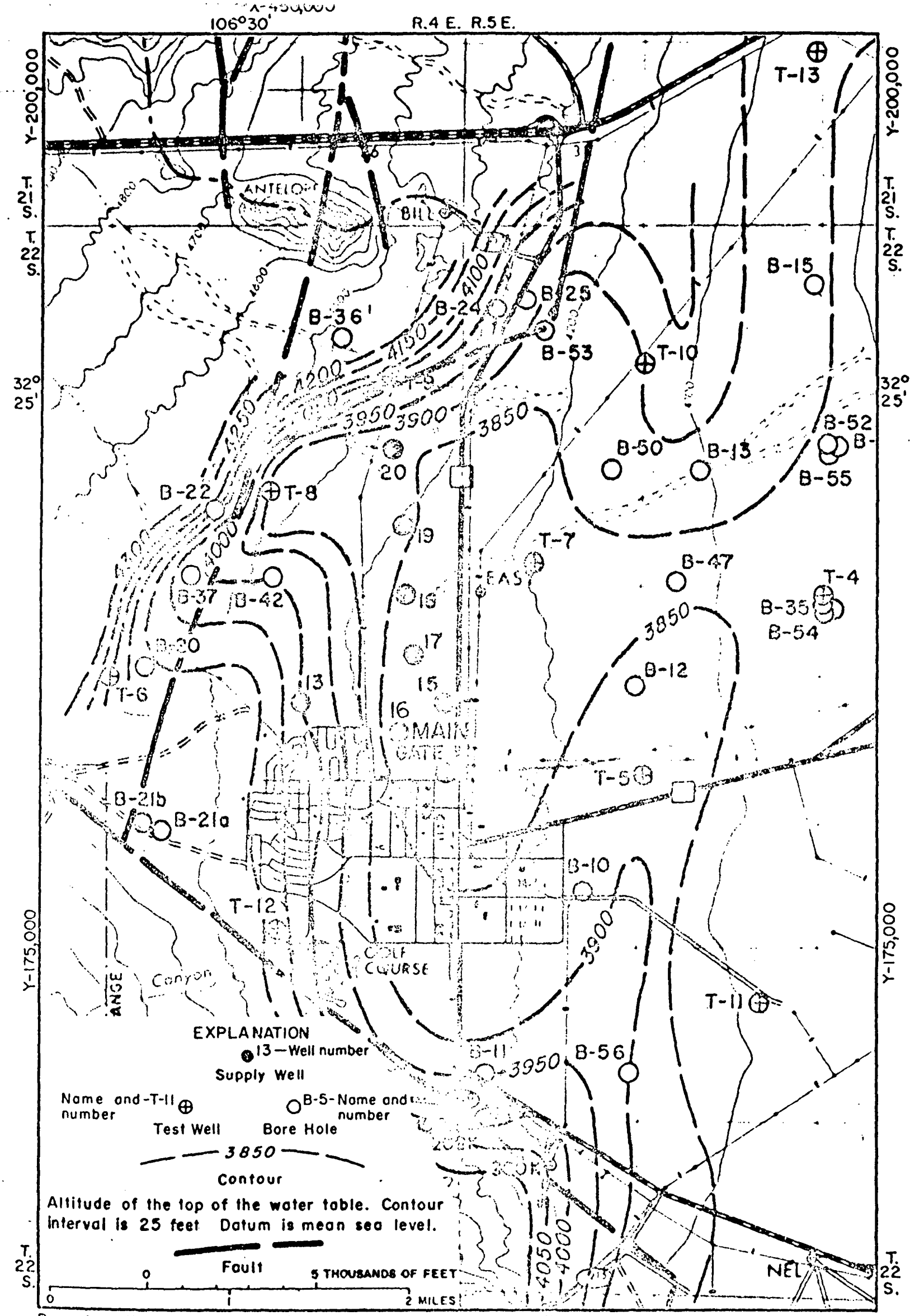

Base from Winite Sands Missile Ronge

Moster Plon Bosic informotion Maps, 1972

Compiled by T.E. Kelly, 1972. Adopled from

IV.C. Ballonce and S.M. Longwill (written commun. 1968)

Figure 26. - Contour map of water table in Post Headquarters area, July 1972 


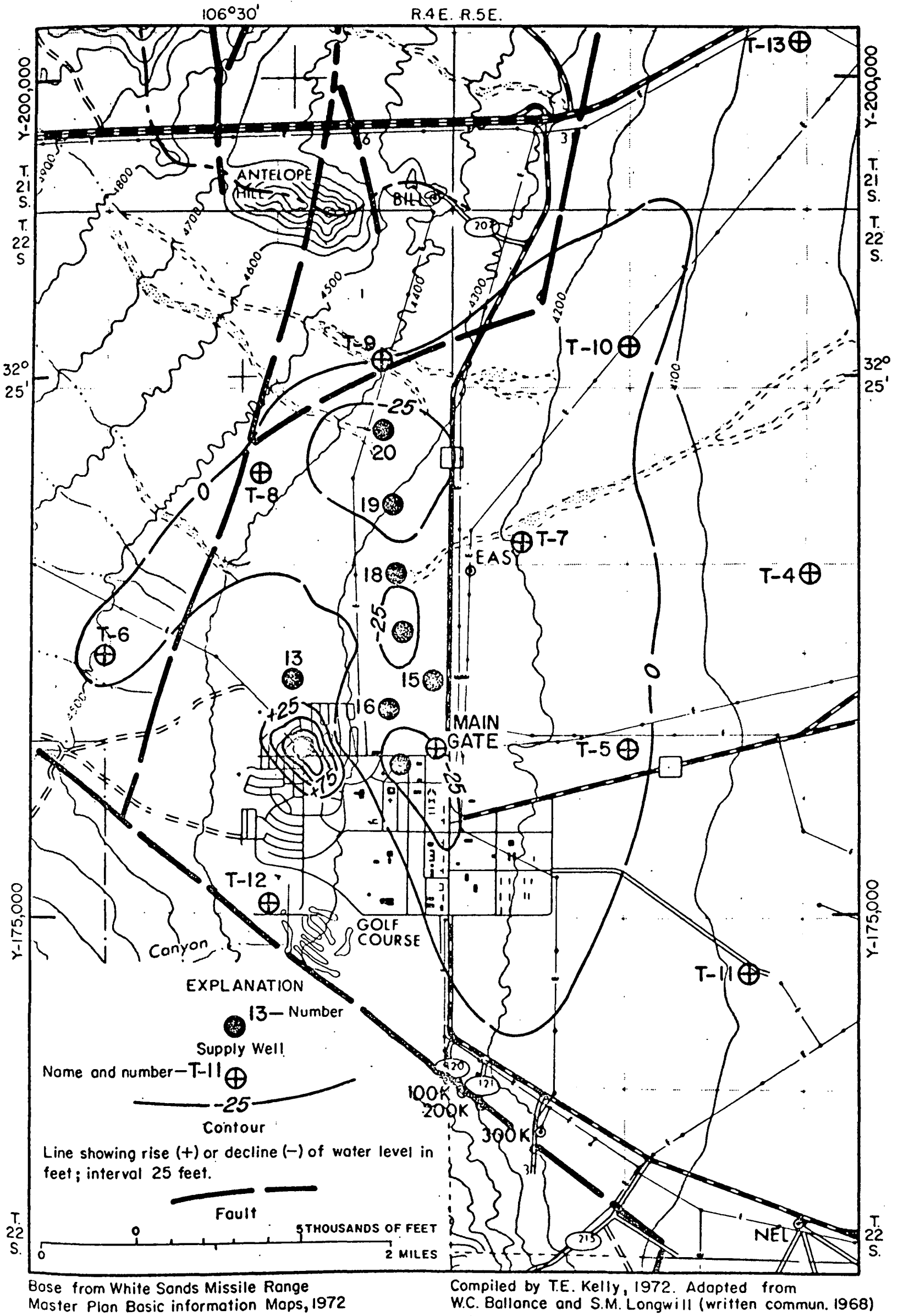

Figure 27.-- Water-level change between 1964 and 1972 . 
The total drawdown of the water table between 1949 and 1972 1s shown in figure 28. This is the combined drawdown illustrated In figures 25 and 27 . The axis of the cone of depression underlies the major producing wells and the area of influence has extended approximately 2 miles east of the well field.

West of the fault zone shown. in figure 29, monzonite is the major geologic unit underlying the permeable clastics containing the fresh-water zone. On the east side of this fault zone, a consolidated conglomerate has been identified beneath the permeable clastics of the fresh-water zone. This conglomerate probably is considerably older than the bolson fill inasmuch as cores from this unit reveal that it is well lithified. Monzonite pebbles derived from nearby mountains comprise a large percentage of the conglomerate, therefore this unit has been difficult to recognize in well cuttings. However, it does have rather prominent characteristics on some geophysical logs. The more mineralized water forms a sloping boundary of the fresh-water zone on the east. These relationships are shown on figure 29.

Figure 30 shows the configuration of the base of the fresh-water zone (less than 1,000 milligrams per liter dissolved solids), and total thickness of the fresh water in the Post Headquarters and adjacent areas is shown in figure 31. This map, based on all data available in July 1972, shows that the maximum thickness is at least 1,500 feet. There is no doubt that the size and configuration of this area is controlled by the Organ Mountain fault zone. The configuration of the water table in July 1972 is shown in figure 32 . 


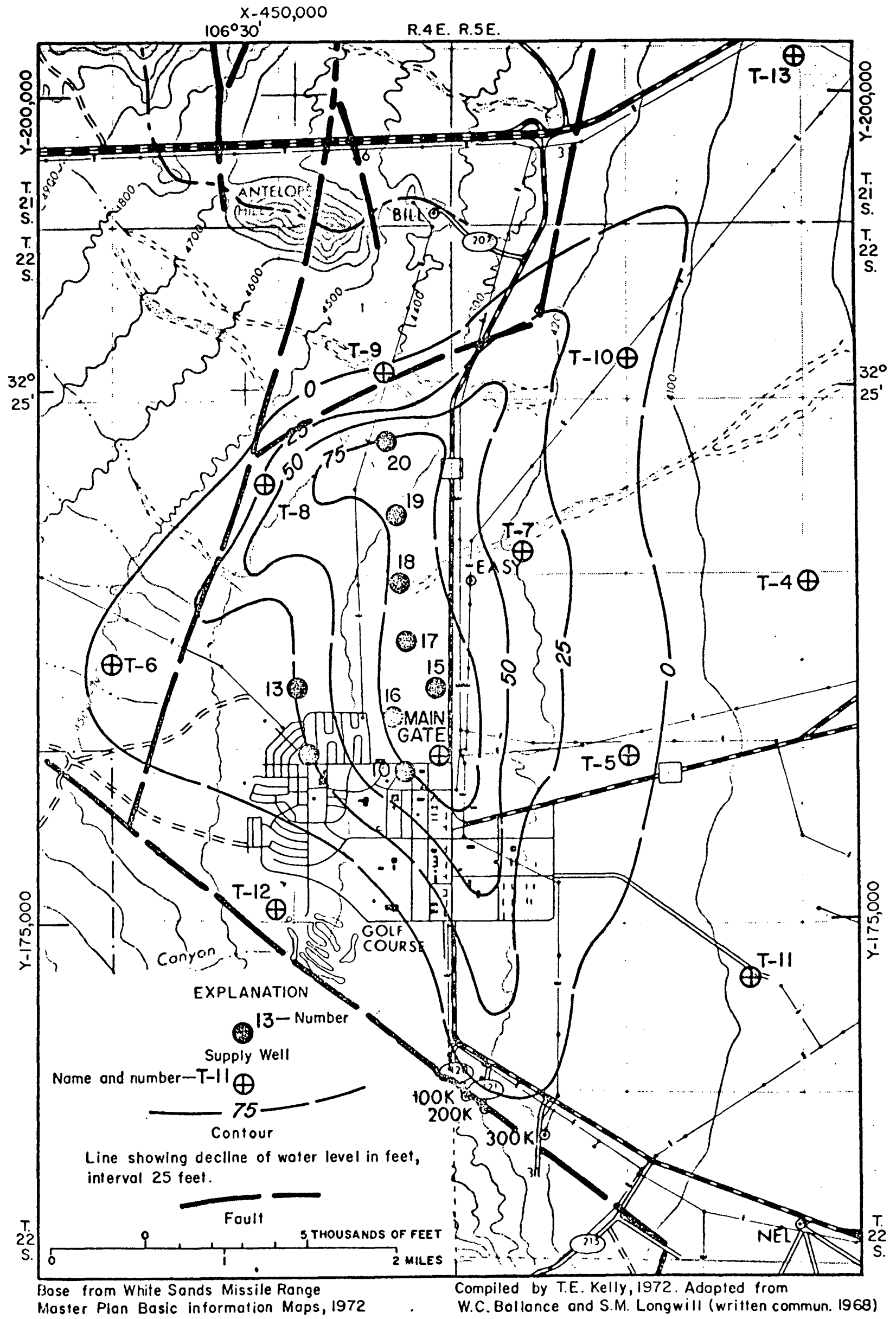

Figure 28.--Water-level change between 1949 and 1972 . 

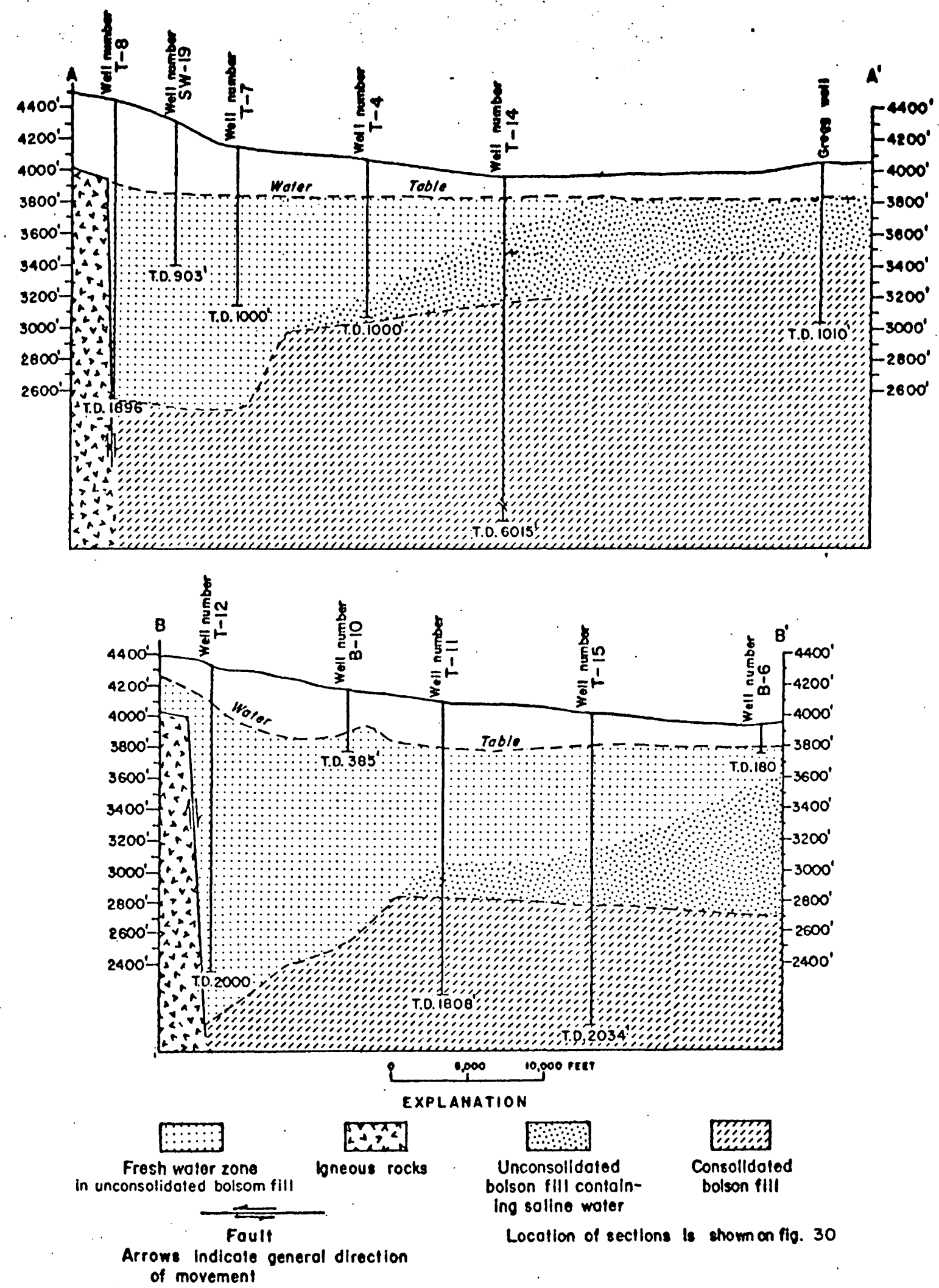

Locotion of sectlons is shomon fig. 30

Figure 29.--Cross sections of the Post Headquarters area. 
A trough of fresh water, having a maximum thickness of about 700 feet, parallels the mountain front north of the Post Headquarters. A similar trough extends southward from the Post Headquarters to the limits of the study area. Throughout the area, fresh water interfaces with saline water along a line which roughly parallels the mountain front.

Water-quality data has been collected during the various ground-water investigations; this is on file in the offices of the U.S. Geological Survey in Albuquerque. Although minor changes in water quality have been noted which deserve continued monitoring, in general there is no evidence to indicate significant intrusion of saline water into the White Sands aquifer. Bar graphs showing the principal chemical constituents in the water from various wells in the project area are illustrated on figure 33 . 


\section{SUMMARY}

Hydrologic data show great variation in transmissivity at different test sites; much of this may be due to well construction and development. Specific capacities based on production figures probably are the most rellable data.

As the well field was expanded northward where greater yields were obtained, water-level declines were distributed more uniformly throughout the aquifer. As much as 100 feet of recovery was recorded in supply well 11 .

The Organ Mountain fault zone is the major controlling factor of the size and configuration of the aquifer; maximum aquifer thickness is 1,500 feet.

In addition to that in the Post Headquarters area, potable water is present in significant quantities along the mountain front; however, hydrologic data are lacking in these areas.

There is considerable variation in the principal chemical constituents found in ground water of the project area.

There is no evidence to indicate that saline-water encroachment has begun in the present well field. 


\section{BIBLIOGRAPHY}

Allen, J.E., and Jones, S. M., 1951, Preliminary stratigraphy and preliminary geologic map, Capitan Quadrangle, Lincoln County, New Mexico, in Roswell Geol. Soc. Guidebook 5 th Field Conf., Capitan, Carrizozo, Chupadera Mesa region, Lincoln and Socorro Counties, New Mexico, 1951: Map and section.

Ballance, W. C., and Basler, J. A., 1967, Runoff from a paved small watershed at White Sands Missile Range, New Mexico: U.S. Geol. Survey open-file report, 21 p., $1 \mathrm{fig}$.

Basler, J. A., 1967, Rehabilitation of wells 13, 15, 16, and 17, Headquarters area, White Sands Missile Range, New Mexico:

U.S. Geol. Survey open-file report, 86 p., 9 figs.

1971, Annual water-resources review, White Sands Missile Range, 1970--a basic-data report: U.S. Geol. Survey open-file report, $33 \mathrm{p}$.

Bjorklund, L. J., 1957, Reconnaissance of ground-water conditions in the Crow Flats area, Otero County, New Mexico: New Mexico State Engineer Tech. Rept. 8, 19 p., 4 figs.

Bodine, M. W., Jr., 1956, Geology of Capitan coal field, Lincoln County, New Mexico: N. Mex. Inst. Min. and Tech., State Bur. Mines and Mineral Res. Div. Circ. 35, 17 p., 3 figs. Busch, F. E., 1969, Annual water-resources review, White Sands Missile Range, 1968--a basic-data report: U.S. Geol. Survey open-file report, $41 \mathrm{p}$. 


\section{BIBLIOGRAPHY - Continued}

Busch, F. E., 1970, Annual water-resources review, White Sands Missile Range, 1969--a basic-data report: U.S. Geol. Survey open-file report, $41 \mathrm{p}$.

Conover, C. S., Herrick, E. H., Hood, J. W., and Weir, J, E., Jr., November 1955, The occurrence of ground water in south-central New Mexico, in New Mexico Geol. Soc. Guidebook 6th Field Conf., South-Central New Mexico, 1955: p. 108-120.

Cooper, J. B., 1970, Summary records of supply wells and test wells In the Post Headquarters area, White Sands Missile Range, New Mexico: U.S. Geol. Survey open-file report, 202 p. Craig, Harmon, 1953, The Geochemistry of the stable isotopes: Geochim. et Cosmochim. Acta, v. 3, p. 52-92.

Cruz, R. R., 1972, Annual water-resources review, White Sands Missile Range, 1971--a basic-data report: U.S. Geol. Survey open-file report, $35 \mathrm{p}$.

Dane, C. H., and Bachman, G. O., 1958, Preliminary Geologic Map of the Southeastern part of New Mexico: U.S. Geol. Survey Map I-256. Darton, N. H., 1922, Geologic structure of parts of New Mexico: U.S. Geol. Survey Bull. 726-E, p. 173-275, 32 figs. -1928, "Red beds" and associated formations in New Mexico: U.S. Geol. Survey Bu11. 794, 356 p., 173 figs. Davis, L. V., and Busch, F. E., 1968, Summary of hydrologic Investigations by the United States Geological Survey at White Sands Missile Range, New Mex1co: U.S. Geol. Survey open-file report, 146 p., 27 figs. 


\section{BIBLIOGRAPHY - Continued}

Doty, G. C., 1963, Water-supply development at the National Aeronautics and Space Agency-Apollo Propulsion System Development Facility, Dona Ana County, N. Mex.: U.S. Geol. Survey open-file report, 40 p., 5 figs.

-1967, Supply well for Dona Ana Range Camp, Dona Ana County, New Mexico: U.S. Geol. Survey open-file report, 23 p.

-1968a, Phase I test wells, White Sands Missile Range, Dona Ana County, New Mexico: U.S. Geol. Survey open-f1le report, 39 p., 12 figs.

-1968b, Summary of wells drilled by White Sands Missije Range from June 1962 to January 1965: U.S. Geol. Survey open-file report, 52 p., 16 figs,

$-1968 c$, Test wells in the Post area, White Sands Missile Range, Dona Ana County, New Mexico: U.S. Geol. Survey open-file report, 29 p., 16 figs.

Doty, G. C., and Cooper, J. B., 1970, Stratigraphic test well T-14, Post area, White Sands Missile Range, Dona Ana County, New Mexico: U.S. Geol. Survey open-file report, 34 p., 7 figs.

Dunham, K. C., 1935, The geology of the Organ Mountains: New Mexico Inst. Mining and Technology, State Bur. Mines and Mineral Resources Div. Bull. 11, p. 272, 21 figs.

Ferris, J. G., Knowles, D. B., Brown, R. H., and Stallman, R. W., 1962, Theory of aquifer tests: U.S. Geol. Survey Water-Supply Paper 1536-E, p. 69-174. 


\section{BIBLIOGRAPHY - Continued}

Follett, C. R., 1954, Records of water-level measurements in

El Paso County, Texas: Texas Board Water Engineers Bull. 5417, $49 \mathrm{p}$

Gardner, J. H., 1910, The Carthage coal field, New Mexico: U.S. Geol. Survey Bull. 381, p. 452-460.

Hale, W. E., Reiland, L. J., and Beverage, J. P., 1965, Characteristics of the Water Supply in New Mexico: New Mexico State Engineer Tech. Rept. 31, $131 \mathrm{p}$.

Hendrickson, G. E., 1949a, Ground-water availability at camp site of Guadalupe Bombing Range, Otero County, New Mexico: U.S. Geol. Survey open-file report, 2 p.

-1949b, Ground-water resources of the Carrizozo area, New Mexico:

U.S. Geol. Survey open-file report, $14 \mathrm{p}$.

Herrick, E. H., 1955, Rehabilitation of wells in the headquarters area, White Sands Missile Range, Dona Ana County, New Mexico: U.S. Geol. Survey open-file report, 26 p., 7 figs.

-1956, Memorandum on a reconnaissance of ground-water conditions southeast of Valmont, Otero County, New Mexico: U.S. Geol. Survey open-file report, 5 p., 1 fig.

-1960a, Ground-water resources of the Headquarters (Cantonment) area, White Sands Proving Ground, Dona Ana County, New Mexico: U.S. Geol, Survey open-file report, 203 p., 33 figs. $-1960 \mathrm{~b}$, Rehabilitation of wells in the Headquarters area, White Sands Proving Ground, Dona Ana County, New Mexico: U.S. Geol. Survey open-file report, 26 p., $7 \mathrm{flgs}$. 


\section{BIBLIOGRAPHY - Continued}

Herrick, E. H., 1961, Conservation of flood water at White Sands Missile Range, Dona Ana County, New Mexico: U.S. Geol. Survey $\mathrm{HA}-42$.

Herrick, E. H., and Davis, L. V., 1965, Avallability of ground water

In Tularosa Basin and adjoining areas, New Mextco and Texas:

U.S. Geol. Survey Hydrol. Inv. Atlas HA-191, 5 p.

Herrick, E. H., and others, 1960, Appraisal of ground-water resources

of Tularosa Basin and adjoining areas, New Mexico and Texas:

U.S. Geol. Survey open-file report, 195 p., 4 figs.

Hood, J. W., 1956a, Ground water in the vicinity of the Atlas site,

Holloman Air Force Base, Otero County, New Mexico: U.S. Geol.

Survey open-file report, 42 p., $4 \mathrm{figs}$.

-1956b, Ground water in the vicinity of McGregor Range Camp Site,

Otero County, New Mexico: U.S. Geol. Survey open-file report,

76 p., 2 figs.

-1956c, Summary of results of ground-water investigations in

the vicinity of Boles well field, Otero County, New Mexico:

U.S. Geol. Survey open-file report, 17 p., 3 figs.

-1956d, Availability of ground water in the vicinity of

Cloudcroft, New Mexico: U.S. Geol. Survey open-file report,

27 p., 2 figs.

1958, Ground-water resources and related geology in the vicinity

of Holloman Air Force Base, Otero County, New Mexico: U.S. Geol.

Survey open-file report, 43 p., 4 figs. 


\section{BIBLIOGRAPHY - Continued}

Hood, J. W., 1959, Summary of results of ground-water Investigations In the vicinity of Boles well fleld, Otero County, New Mexico: U.S. Geol. Survey open-f1le report, 17 p., 3 figs.

-1960, Ground water in the vicinity of the Atlas site, Holloman Alr Force Base, Otero County, New Mexico: U.S. Geol. Survey openfile report, 38 p., 4 figs.

1968, Ground-water investigations at White Sands Missile Range, New Mexico, July 1960-June 1962: U.S. Geol. Survey open-file report, 153 p., 28 figs.

Hood, J. W., and Herrick, E. H., 1962, Water resources of the Three Rivers area, Otero and Lincoln Counties, New Mexico: U.S. Geol. Survey open-file report, 23 p., 3 figs.

Kelley, V. C., 1955, Geologic map of the Sierra County region, New Mexico, in New Mexico Geol. Soc. Guidebook 6th Field Conf., 1955.

Kelley, V. C., and Silver, Caswell, 1952, Geology of the Caballo Mountains [New Mexico]: New Mexico Univ. Pub., Geol. Ser., no. $4,286 \mathrm{p}$.

Kelly, T. E., Myers, B. N., and Hershey, L. A., 1970, Saline groundwater resources of the Rio Grande drainage basin--a pilot study: Dept. of Interior, U.S. Office of Saline Water Research and Devel. Prog. Rept. 260, 71 p.

Keyes, C. R., 1905, Geology and underground water conditions of the Jornada del Muerto, New Mexico: U.S. Geol. Survey Water-Supply Paper 123, 42 p., map. 


\section{BIBLIOGRAPHY - ContInued}

King, P. B., 1948; Geology of the southern Guadalupe Mountains, Texas: U.S. Geo1. Survey Prof. Paper 215, 173 p., 24 figs.

-1949, Regional geologic map of parts of Culbertson and Hudspeth Counties, Texas: U.S. Geol. Survey 011 and Gas Inv. Prelim. Map 90.

Knowles, D. B., and Kennedy, R. A., Ground-water resources of the Hueco Bolson northeast of E1 Paso, Texas: U.S. Geol. Survey WaterSupply Paper 1426, 183 p., 14 figs.

Kottlowski, F. E., Flower, R. H., Thompson, M. L., and Foster, R. W., 1956, Stratigraphic studies of the San Andres Mountains, New Mexico: N. Mex. Inst, Min, and Tech., State Bur. Mines and Mineral Res. Memo. 1, 132 p.

Laudon, L. R., 1949, Mississippian formations of southwestern New Mexico: Geol. Soc. America Bul1., v. 60, no. 1, p. 13-14, fig. 4 .

Laudon, L. R., and Bowsher, A. L., 1941, Mississippian formations of Sacramento Mountains, New Mexico: Am. Assoc. Petroleum Geologists Bul1., v. 25, no. 12, p. 2107-2160.

Leatherwood, F. N., and Peterson, D. F., Jr., 1954, Hydraulic head loss at the interface between uniform sands of different sizes: Am. Geophys. Union Trans., p. 588-594.

Lee, W. T., 1907, Water resources of the Rio Grande valley in New Mexico and their development: U.S. Geol. Survey Water-Supply Paper 188, $59 \mathrm{p}$. 


\section{BIBLIOGRAPHY - Continued}

Leggat, E. R., 1957, Memorandum on the water-supply wells at BIggs Alr Force Base, E1 Paso, Texas: U.S. Geol. Survey open-file report, 8 p., 4 figs.

Lindgren, Waldemar, Graton, L. C., and Gordon, C. H., 1910, The ore deposits of New Mexico: U.S. Geol. Survey Prof. Paper 68, 361 p. Lyford, F. P., 1970a, Test we11s T-15, T-16, T-17, T-18, and RC-3, White Sands Missile Range, Dona Ana and Socorro Counties, New Mexico: U.S. Geo1. Survey open-file report, 44 p., 14 figs. $-1970 \mathrm{~b}$, Test we11s T-15, T-16, T-17, T-18, and RC-3, White Sands Missile Range, Dona Ana and Socorro Counties, New Mexico: U.S. Geol. Survey open-file report, 46 p., 14 figs.

$-1970 \mathrm{c}$, Water supply we11 SRC-2, Stallion Range Center, White Sands Missile Range, Socorro County, New Mexico: U.S. Geol. Survey open-file report, 26 p., 8 figs.

McLean, J. S., 1970, Saline ground-water resources of the Tularosa basin, New Mexico: Dept. of Interior, U.S. Office of Saline Water Research and Devel. Prog, Rept. 561, 128 p.

Meeks, T. 0., 1950, The occurrence of ground water in the AlamogordoTularosa area of the Otero Soll Conservation District, New Mexico: U.S. Dept. Agriculture, Soil Conserv. Service Regional Bull. 111, Geo1. Ser. 2, 42 p., 2 figs.

Meinzer, 0. E., 1923, Outline of ground-water hydrology: U.S. Geol. Survey Water-Supply Paper 494, $71 \mathrm{p}$.

-1932, Outline of methods for estimating ground-water supplies: U.S. Geol. Survey Water-Supply Paper 638-C, p. 99-144. 


\section{BIBLIOGRAPHY - ContInued}

Meinzer, 0. E., and Hare, R. F., 1915, Geology and water resources of Tularosa Basin, N. Mex.: U.S. Geo1. Survey Water-Supply Paper 343, 317 p., maps.

Mourant, W. A., 1957, Reconnalssance of water resources in the upper

- part of Sacramento River canyon, New Mexico: U.S. Geol. Survey open-file report, 25 p., 3 figs.

-1.959, Reconnaissance of water resources in the upper part of Sacramento River canyon, New Mexico: U.S. Geol. Survey open-file report, 25 p., 3 figs.

Murray, C. R., 1954, Memorandum on the possibilities of developing ground water for the Alamogordo Army Air Base, Otero County, New Mexico: U.S. Geol. Survey open-file report, 4 p. Needham, C. E., and Bates, R. L., 1943, Permian type sections in central New Mexico: Geol. Soc. America Bul1., v. 54, p. 1653-1667. New Mexico Geological Society, 1.955, Guidebook of southcentral New Mexico: New Mexico Geol. Soc. 6th Field Conf., 182 p. New Mexico State Engineer Office, 1956, Climatological summary, New Mexico, Precipitation, 1949-1954: New Mexico State Engineer Tech. Rept. 6, $407 \mathrm{p}$.

Pearson, F. J., Jr., and White, D. E., 1967, Carbon-14 ages and flow rates of water in Carrizo Sand, Atacosa County, Texas: Water Resources Research, v. 3, no. 1, p. 251-261. 


\section{BIBLIOGRAPHY - Continued}

Pray, L. C., 1952, Stratigraphy of the escarpment of the Sacramento Mountains, Otero County, New Mexico: Ms, rept, on file at $\mathrm{N}$. Mex. Inst. Min. and Tech., State Bur. Mines and Mineral Res. Div., Socorro, N. Mex., 361 p., 48, figs.

1954, Outline of the stratigraphy and structure of the Sacramento Mountains escarpment [New Mexico], in Guidebook of southeastern New Mexico: New Mexico Geo1. Soc. Guidebook 5th Field Conf., p. 92-107.

Public Health Service drinking water standards, 1946, Federa1 Security Agency, Public Health Service: Public Health Repts., v. 61 , no. 11, p. $371-384$.

Rapp, J. R., 1957, Summary of test drilling and ground-water conditions in the McGregor Range area, Otero and Dona Ana Counties, New Mexico, and El Paso County, Texas: U.S. Geol. Survey open-file report, $22 \mathrm{p}$.

Reeder, H. O., Berkstresser, C. F., Jr., Mower, R. W., Herrick, E. H., Doty, G. C., Bjorklund, L. J., Hood, J. W., and Gordon, E. D., 1959, Annual water-level measurements in observation wel1s, 1951-1955, and atlas of maps showing changes in water levels for various periods from beginning of record through 1954, New Mexico: New Mexico State Engineer Tech. Rept. 13, 339 p., 4 figs. 


\section{BIBLIOGRAPHY - Continued}

Reeder, H. 0., Doty, G. C., Cooper, J. B., Mower, R. W., Bjorklund, L. J., Busch, F, E., Benge, U. N., Mourant, W. A., 1960, Ground-water levels in New Mexico, 1956: New Mexico State Engineer Tech. Rept. 19, 251 p., 19 figs.

Reeder, H. 0., Doty, G. C., Mower, R. W., Bjorklund, L. J., Benge, U. N., Hood, J. W., Busch, F. E., and Berkstresser, C. F., $\mathrm{Jr} ., 1960$, Changes in water levels in 1955 and annual water-level measurements in January and February 1956 in observation wells in New Mexico: New Mexico State Engineer Tech. Rept. 16, 145 p., 31 figs.

Reeder, H. O., Doty, G. C., Mower, R. W., Cooper, J. B., Cox, E. R., Mourant, W. A., Busch, F. E., Benge, U. N, , and Maxwell, B. W., 1961, Ground-water levels in New Mexico, 1957: New Mexico State Engineer Tech. Rept. 22, 306 p.

Sayre, A. N., and Livingston, Penn, 1945, Ground-water resources of the El Paso area, Texas: U.S. Geol. Survey Water-Supply Paper 919, $188 \mathrm{p.,} 11 \mathrm{figs}$.

Scalapino, R. A., 1949, Ground-water resources of the E1 Paso area, Texas; Prog. Rept. 6: Texas Board Water Engineers, 31 p., 10 figs. Scott, A. G., 1970, Estimated mean-annual runoff at Post Headquarters area, White Sands Missile Range, New Mexico: U.S. Geol. Survey open-file report, $13 \mathrm{p}$.

Smith, R. E., 1956, Ground-water resources of the E1 Paso area, Texas; Prog. Rept. 7: Texas Board Water Engineers Bull. 5603, 33 p., $6 \mathrm{figs.}$ 


\section{BIBLIOGRAPHY - ContInued}

Stevenson, F. W., 1945, Devonian of New Mexico: Jour. Geology, v. 53 , no. 4 , p. $217-245$,

Sundstrom, R. W., and Hood, J, W., 1952, Results of artificial recharge of the ground-water resources at El Paso, Texas: Texas Board Water Engineers Bull. 5206, 19 p., 7 flgs.

Theis, C. V., 1935, The relation between the lowering of the piezometric surface and the rate and duration of discharge of a well using ground-water storage: Am. Geophys. Union Trans., pt. 2, v. 16, p. 519-534; U.S. Geol. Survey Ground-Water Note 5, 9 p., [August 1952].

-1937, Amount of ground-water recharge in the Southern High Plains: Am. Geophys. Union Trans., p. 564-568. 1938, The significance and nature of the cone of depression in ground-water bodies: Econ. Geol., v. XXXIII, no. 8, p. 889-902.

-1940, The source of water derived from wells: Civil Eng., v. 10 , no. 5, p. $277-280$.

U.S. Salinity Laboratory Staff, Richards, L. A., ed., 1954, Diagnosis and improvement of saline and alkali soils: U.S. Dept. Agriculture, Handbook No. 60 .

U.S. Public Health Service, 1946, Drinking water standards: Public Health Repts., v. 61, no. 11, p. 371-384. Weir, J. E., Jr., 1965, Geology and availability of ground water in the northern part of the White Sands Missile Range and vicinity, New Mexico: U.S. Geol. Survey Water-Supply Paper 1801, 78 p. 


\section{BIBLIOGRAPHY - Concluded}

Wenzel, L. K., 1942, Methods for determining permeability of waterbearing materials, with special reference to discharging well methods: U.S. Geol. Survey Water-Supply Paper 887, 192 p. Wilpolt, R. H., and Wanek, A. A., 1951, Geology of the region from Socorro and San Antonio east to Chupadera Mesa, Socorro County, New Mexico: U.S. Geol. Survey 011 and Gas Inv. Map OM-121, 2 sheets.

Zohdy, A. A. R., Jackson, D. B., Mattick, R. E, , and Peterson, D. L., 1969, Geophysical surveys for ground water at White Sands Missile Range, New Mexico: U.S. Geol. Survey open-file report, 144 p., 17 figs. 